\title{
Infinitely Many Solutions for a Class of Fractional Impulsive Coupled Systems with $(p, q)$-Laplacian
}

\author{
Junping Xie $\mathbb{D}^{1,2}$ and Xingyong Zhang $\mathbb{D}^{3,4}$ \\ ${ }^{1}$ School of Geosciences and Info-Physics, Central South University, Changsha, Hunan 410083, China \\ ${ }^{2}$ Key Laboratory of Metallogenic Prediction of Nonferrous Metals and Geological Environment Monitoring, Central South University, \\ Ministry of Education, Changsha, China \\ ${ }^{3}$ Faculty of Science, Kunming University of Science and Technology, Kunming, Yunnan 650500, China \\ ${ }^{4}$ School of Mathematics and Statistics, Central South University, Changsha, Hunan 410083, China
}

Correspondence should be addressed to Xingyong Zhang; zhangxingyong1@163.com

Received 23 January 2018; Accepted 28 March 2018; Published 8 May 2018

Academic Editor: Antonio Iannizzotto

Copyright (C) 2018 Junping Xie and Xingyong Zhang. This is an open access article distributed under the Creative Commons Attribution License, which permits unrestricted use, distribution, and reproduction in any medium, provided the original work is properly cited.

By using the symmetric mountain pass lemma, we investigate the problem of existence of infinitely many solutions for a class of fractional impulsive coupled systems with $(p, q)$-Laplacian, which possesses mixed type nonlinearities, and the nonlinearities do not need to satisfy the well-known Ambrosetti-Rabinowitz condition.

\section{Introduction and Main Results}

In this paper, we are concerned with existence of infinitely many solutions for the following fractional impulsive differential system with $(p, q)$-Laplacian:

$$
\begin{aligned}
{ }_{t} D_{T}^{\alpha}\left(\rho(t) \Phi_{p}\left({ }_{0}^{c} D_{t}^{\alpha} u(t)\right)\right) & =\nabla_{u} W(t, u(t), v(t)), \\
\text { a.e. } t \in[0, T], & \\
{ }_{t} D_{T}^{\beta}\left(\gamma(t) \Phi_{q}\left({ }_{0}^{c} D_{t}^{\beta} v(t)\right)\right) & =\nabla_{v} W(t, u(t), v(t)), \\
\text { a.e. } t \in[0, T], & \quad i=1,2, \ldots, l, \\
\Delta\left({ }_{t} D_{T}^{\alpha-1}\left(\rho \Phi_{p}\left({ }_{0}^{c} D_{t}^{\alpha} u\right)\right)\right)\left(t_{i}\right) & =\nabla I_{i}\left(u\left(t_{i}\right)\right), \\
\Delta\left({ }_{t} D_{T}^{\beta-1}\left(\gamma \Phi_{q}\left({ }_{0}^{c} D_{t}^{\beta} v\right)\right)\right)\left(s_{j}\right) & =\nabla H_{j}\left(v\left(s_{j}\right)\right), \\
& j=1,2, \ldots, m, \\
u(0) & =u(T)=0, \\
v(0) & =v(T)=0,
\end{aligned}
$$

where $T>0, \alpha \in(1 / p, 1]$ with $p>1, \beta \in(1 / q, 1]$ with $q>1, \Phi_{s}(x)=|x|^{s-2} x(s>1$ and $s=p, q)$, and ${ }_{t} D_{T}^{\alpha}$ (or ${ }_{t} D_{T}^{\beta}$ ) denotes the right Riemann-Liouville fractional derivative of order $\alpha$ (or $\beta),{ }_{0}^{c} D_{t}^{\alpha}$ (or ${ }_{0}^{c} D_{t}^{\beta}$ ) is the left Caputo fractional derivative of order $\alpha$ (or $\beta), \rho, \gamma \in L^{\infty}\left([0, T], \mathbb{R}^{+}\right)$, $I_{i}, H_{j}: \mathbb{R}^{N}(N \geq 1) \rightarrow \mathbb{R}$ are continuously differentiable, $0=t_{0}<t_{1}<\cdots<t_{l+1}=T, 0=s_{0}<s_{1}<\cdots<s_{m+1}=T$, and

$$
\begin{gathered}
\Delta\left({ }_{t} D_{T}^{\alpha-1}\left(\rho \Phi_{p}\left({ }_{0}^{c} D_{t}^{\alpha} u\right)\right)\right)\left(t_{i}\right) \\
={ }_{t} D_{T}^{\alpha-1}\left(\rho \Phi_{p}\left({ }_{0}^{c} D_{t}^{\alpha} u\right)\right)\left(t_{i}^{+}\right) \\
\quad-{ }_{t} D_{T}^{\alpha-1}\left(\rho \Phi_{p}\left({ }_{0}^{c} D_{t}^{\alpha} u\right)\right)\left(t_{i}^{-}\right), \\
\Delta\left({ }_{t} D_{T}^{\beta-1}\left(\gamma \Phi_{q}\left({ }_{0}^{c} D_{t}^{\beta} v\right)\right)\right)\left(s_{j}\right) \\
={ }_{t} D_{T}^{\beta-1}\left(\gamma \Phi_{q}\left({ }_{0}^{c} D_{t}^{\beta} v\right)\right)\left(s_{j}^{+}\right) \\
-{ }_{t} D_{T}^{\beta-1}\left(\gamma \Phi_{q}\left({ }_{0}^{c} D_{t}^{\beta} v\right)\right)\left(s_{j}^{-}\right),
\end{gathered}
$$


where

$$
\begin{aligned}
& { }_{t} D_{T}^{\alpha-1}\left(\rho \Phi_{p}\left({ }_{0}^{c} D_{t}^{\alpha} u\right)\right)\left(t_{i}^{+}\right) \\
& =\lim _{t \rightarrow t_{i}^{+}} D_{T}^{\alpha-1}\left(\rho \Phi_{p}\left({ }_{0}^{c} D_{t}^{\alpha} u\right)\right)(t), \\
& { }_{t} D_{T}^{\alpha-1}\left(\rho \Phi_{p}\left({ }_{0}^{c} D_{t}^{\alpha} u\right)\right)\left(t_{i}^{-}\right) \\
& \quad=\lim _{t \rightarrow t_{i}^{-}} D_{T}^{\alpha-1}\left(\rho \Phi_{p}\left({ }_{0}^{c} D_{t}^{\alpha} u\right)\right)(t), \\
& { }_{t} D_{T}^{\beta-1}\left(\gamma \Phi_{q}\left({ }_{0}^{c} D_{t}^{\beta} v\right)\right)\left(s_{j}^{+}\right) \\
& \quad=\lim _{s \rightarrow s_{j}^{+}} D_{T}^{\beta-1}\left(\gamma \Phi_{q}\left({ }_{0}^{c} D_{t}^{\beta} v\right)\right)(s), \\
& { }_{t} D_{T}^{\alpha-1}\left(\gamma \Phi_{q}\left({ }_{0}^{c} D_{t}^{\beta} v\right)\right)\left(s_{j}^{-}\right) \\
& =\lim _{s \rightarrow s_{j}^{-}} D_{T}^{\beta-1}\left(\gamma \Phi_{q}\left({ }_{0}^{c} D_{t}^{\beta} v\right)\right)(s),
\end{aligned}
$$

$i=1,2, \ldots, l, j=1,2, \ldots, m$, and $W:[0, T] \times \mathbb{R}^{N} \times \mathbb{R}^{N} \rightarrow \mathbb{R}$ satisfies $W(t, x, y)=-K(t, x, y)+F(t, x, y)$ and the following assumptions.

(W0) $W(t, x, y)$ is measurable in $t$ for each $(x, y) \in \mathbb{R}^{N} \times$ $\mathbb{R}^{N}$, continuously differentiable in $(x, y) \in \mathbb{R}^{N} \times \mathbb{R}^{N}$ for a.e. $t \in[0, T]$, and there exist $a_{1}, a_{2} \in C\left(\mathbb{R}^{+}, \mathbb{R}^{+}\right)$and $b \in$ $L^{\infty}\left([0, T] ; \mathbb{R}^{+}\right)$such that

$$
\begin{aligned}
& |K(t, x, y)|+|\nabla K(t, x, y)|+|F(t, x, y)| \\
& \quad+|\nabla F(t, x, y)| \leq\left[a_{1}(|x|)+a_{2}(|y|)\right] b(t), \\
& \left|I_{i}(x)\right|+\left|\nabla I_{i}(x)\right| \leq a_{1}(|x|), \quad i=1,2, \ldots, l, \\
& \left|H_{j}(y)\right|+\left|\nabla H_{j}(y)\right| \leq a_{2}(|y|), \quad j=1,2, \ldots, m
\end{aligned}
$$

for all $(x, y) \in \mathbb{R}^{N} \times \mathbb{R}^{N}$ and a.e. $t \in[0, T]$.

It is well known that critical point theory is a very important and effective tool to investigate the existence and multiplicity of various solutions for partial differential equations, ordinary differential equations, Hamiltonian systems, difference equations, and so on. Lots of important and interesting results have been established (see, e.g., [1-11] and reference therein). In 2011, Jiao and Zhou [12] first used critical point theory to investigate the existence of solutions for a class of fractional boundary value problems. Since then, critical point theory has also become an effective tool to obtain the existence and multiplicity results of solutions for various fractional differential equations (see, e.g., [13-17] and reference therein). Particularly, in [14], Zhao et al. considered existence of solutions for the following fractional coupled differential system with a parameter:

$$
\begin{aligned}
{ }_{t} D_{T}^{\alpha}\left(a(t){ }_{0} D_{t}^{\alpha} u(t)\right) & =\lambda \nabla_{u} F(t, u(t), v(t)), \\
{ }_{t} D_{T}^{\beta}\left(b(t){ }_{0} D_{t}^{\beta} v(t)\right) & =\lambda \nabla_{v} F(t, u(t), v(t)), \\
t \in(0, T), & \\
u(0) & =u(T)=0, \\
v(0) & =v(T)=0,
\end{aligned}
$$

where $\lambda>0$ is a parameter, $a, b \in L^{\infty}\left([0, T] ; \mathbb{R}^{+}\right)$, and $F:[0, T] \times \mathbb{R}^{2} \rightarrow \mathbb{R}$. By using a critical point theorem in [18], they obtained system (5) which has at least three weak solutions. In [13], Li et al. investigated a class of fractional coupled differential systems with a parameter:

$$
\begin{aligned}
{ }_{t} D_{T}^{\alpha}\left(a(t){ }_{0} D_{t}^{\alpha} u(t)\right) & =\lambda v(t)+\nabla_{u} F(t, u(t), v(t)), \\
t \in(0, T), & \\
{ }_{t} D_{T}^{\beta}\left(b(t){ }_{0} D_{t}^{\beta} v(t)\right) & =\lambda u(t)+\nabla_{v} F(t, u(t), v(t)), \\
t \in(0, T), & \\
u(0) & =u(T)=0, \\
v(0) & =v(T)=0,
\end{aligned}
$$

where $\lambda>0$ is a parameter, $a, b \in L^{\infty}\left([0, T] ; \mathbb{R}^{+}\right)$, and $F:[0, T] \times \mathbb{R}^{2} \rightarrow \mathbb{R}$. By using the least action principle and symmetric mountain pass theorem, they obtained system (6) which has at least one solution under asymptotically quadratic case and has infinitely many solutions under superquadratic case. For the superquadratic case, they assumed the following well-known Ambrosetti-Rabinowitz (AR) condition.

(AR) There are constants $\mu>2, M>0$ such that

$$
0<\mu F(t, x, y) \leq x F_{x}(t, x, y)+y F_{y}(t, x, y)
$$

for all $t \in[0, T]$ and $|x|+|y| \geq M$.

Over the past ten years, integer order impulsive differential equations with different boundary value conditions have been investigated deeply via variational methods (e.g., see [19-26] and reference therein). Recently, Bonanno et al. [27] and Rodrìguez-López and Tersian [28] were concerned with the following second-order impulsive fractional differential equation:

$$
\begin{array}{rl}
{ }_{t} D_{T}^{\alpha}\left({ }_{0}^{c} D_{t}^{\alpha} u(t)\right)+a(t) u(t) & =\lambda f(t, u(t)), \\
t \neq t_{i}, & \text { a.e. } t \in[0, T], \\
\Delta\left({ }_{t} D_{T}^{\alpha-1}\left({ }_{0}^{c} D_{t}^{\alpha} u\right)\right)\left(t_{i}\right) & =\mu Q_{i}\left(u\left(t_{i}\right)\right), \\
i & i=1,2, \ldots, l, \\
u(0) & =u(T)=0,
\end{array}
$$

where $\alpha \in(1 / 2,1], \lambda \in(0,+\infty)$ and $\mu \in(0,+\infty)$ are two parameters, $f \in C([0, T] \times \mathbb{R}, \mathbb{R}), Q_{i} \in C(\mathbb{R}, \mathbb{R})$, and $a \in C([0, T], \mathbb{R})$. By using variational methods, they obtained some existence results about one or three solutions of (8). Subsequently, in [29], Nyamoradi and RodríguezLópez investigated the existence and multiplicity of solutions for (8) with $\lambda=\mu=1$. They obtained some existence results about one or infinitely many solutions of (8) by using the least action principle, the mountain pass theorem, and the symmetric mountain pass theorem. In [30],Y. Zhao and Y. Zhao investigated the existence and multiplicity of 
solutions for a class of perturbed fractional differential system with impulsive effects and one parameter, and they obtained system that has at least one or two nontrivial solutions by using two abstract critical point theorems due to [31]. In [32], Heidarkhani et al. investigated the multiplicity of solutions for a class of perturbed fractional differential system with impulsive effects and two parameters, and they obtained that system has infinitely many solutions by using the smooth version of an abstract critical point theorem due to [33]. In [34], Zhao et al. investigated the existence of solution for (8) with $\lambda=\mu=1$. By using the Morse theory and local linking argument, they obtained that equation has at least one nontrivial solution.

In [35], Zhao and Tang investigated the following impulsive fractional differential equations with $p$-Laplacian:

$$
\begin{aligned}
&{ }_{t} D_{T}^{\alpha}\left(\Phi_{p}\left({ }_{0}^{c} D_{t}^{\alpha} u(t)\right)\right)+|u(t)|^{p-2} u(t)=f(t, u(t)), \\
& t \in(0, T), \\
& \Delta\left({ }_{t} D_{T}^{\alpha-1}\left(\Phi_{p}\left({ }_{0}^{c} D_{t}^{\alpha} u\right)\right)\right)\left(t_{i}\right)= Q_{i}\left(u\left(t_{i}\right)\right), \\
& i=1,2, \ldots, l, \\
& u(0)=u(T)=0,
\end{aligned}
$$

where $f \in C([0, T] \times \mathbb{R}, \mathbb{R})$ and $Q_{i} \in C(\mathbb{R}, \mathbb{R})$. By using the mountain pass theorem, a critical point theorem in [36], and symmetric mountain pass theorem, they obtained two multiplicity results of solutions for (9). In detail, they obtained the following theorems.

Theorem A (see [35]). Suppose the following conditions hold.

(A1) There exists a constant $\mu>p$ such that $Q_{i}(u) u \leq$ $\mu \int_{0}^{u} Q_{i}(s) d s<0$ for any $u \in E^{\alpha, p} \backslash\{0\}, i=1,2, \ldots, l$, where $E_{0}^{\alpha, p}$ is defined in Section 2.

$(A 2)$ There exists a constant $\theta \in(p, \mu]$ such that $\theta F(t, u) \leq$ $f(t, u) u$ for all $u \in E_{0}^{\alpha, p}$ and $t \in[0, T]$, where $F(t, u)=$ $\int_{0}^{u} f(t, s) d s$.

(A3) There exist constants $\delta, \gamma>0$ such that $F^{0} \leq \delta$ and $F_{\infty} \geq \gamma$, where

$$
\begin{gathered}
F^{0}=\limsup _{|u| \rightarrow 0} \frac{F(t, u)}{|u|^{\theta}}, \\
F_{\infty}=\liminf _{|u| \rightarrow \infty} \frac{F(t, u)}{|u|^{\theta}} .
\end{gathered}
$$

(A4) There exist constants $\delta_{i}>0$ such that $\int_{0}^{u} Q_{i}(s) d s \geq$ $-\delta_{i}|u|^{\mu}$ for all $u \in E^{\alpha, p} \backslash\{0\}, i=1,2, \ldots, l$.

Then (9) has at least two weak solutions.

Theorem B (see [35]). Suppose (A1)-(A4) hold and $f(t, u)$ and $I_{i}(u)$ are odd about $u$, where $i=1, \ldots, l$. Then (9) has infinitely many weak solutions.

Motivated by $[12-14,35]$, in this paper, we investigate the existence of infinitely many solutions for system (1). Obviously, system (1) is more general and complex than system
(5), system (6), and (9). We present some techniques in [35], which were applied to fractional $p$-Laplacian impulsive differential equation and can also be applied to fractional $(p, q)$-Laplacian impulsive differential system, and present some more relaxed superquadratic conditions for nonlinearities than those in [35]. It is remarkable that the fractional coupled $(p, q)$-Laplacian differential systems are different from the fractional $p$-Laplacian differential equations. One stark difference is that the solutions of system (1) are the combination of $u_{1}$ and $u_{2}$ but not of (5), which causes the fact that system (1) number is possibly more than that of (9) and, hence, it is impossible that system (1) reduces to system (9). Moreover, since, in general, $p \neq q$ and we present more relaxed superquadratic conditions, it is difficult to prove the boundness of Cerami sequence (see the definition in Section 2 below) and we have to develop some techniques on inequalities. When $\alpha=\beta=1$, system (1) becomes the following integer order $(p, q)$-Laplacian impulsive differential system:

$$
\begin{aligned}
\frac{d}{d t}\left(\rho(t) \Phi_{p}(\dot{u}(t))\right) & =\nabla_{u} W(t, u(t), v(t)), \\
\text { a.e. } t \in[0, T], & \text { a.e. } t \in[0, T], \\
\frac{d}{d t}\left(\gamma(t) \Phi_{q}(\dot{v}(t))\right) & =\nabla_{v} W(t, u(t), v(t)), \\
\Delta\left(\rho \Phi_{p}(\dot{u}(t))\right)\left(t_{i}\right) & =\nabla I_{i}\left(u\left(t_{i}\right)\right), \quad i=1,2, \ldots, l, \\
\Delta\left(\gamma \Phi_{q}(\dot{u}(t))\right)\left(s_{j}\right) & =\nabla H_{j}\left(v\left(s_{j}\right)\right), \\
u(0) & =u(T)=0, \quad j=1,2, \ldots, m, \\
v(0) & =v(T)=0 .
\end{aligned}
$$

There have been some results on existence and multiplicity of solutions for integer order $(p, q)$-Laplacian impulsive differential systems with different boundary value conditions (see, e.g., $[4,37,38])$. However, system (11) which has Dirichlet boundary value is different from those systems in $[4,37,38]$ and our assumptions on $W$ are more relaxed than the wellknown (AR) condition. Hence, our results are still new for integer order $(p, q)$-Laplacian impulsive differential systems. Next, we state our results.

Theorem 1. Suppose that (W0) the following conditions hold. $(\mathscr{A}) \rho^{-}:=\operatorname{essinf}_{[0, T]} \rho(t)>0, \gamma^{-}:=\operatorname{essinf}_{[0, T]} \gamma(t)>0$.

$(W 1) W(t, x, y)$ is even in $(x, y) \in \mathbb{R}^{N} \times \mathbb{R}^{N}$ and $W(t, 0,0) \equiv 0$ for a.e. $t \in[0, T]$.

(K1) There exist constants $\theta_{1} \in[0, p) \theta_{2} \in[0, q), d_{1}, d_{2}>$ $0, M_{1}>0$ such that

$$
K(t, x, y) \geq-d_{1}|x|^{\theta_{1}}-d_{2}|y|^{\theta_{2}}
$$

for a.e. $t \in[0, T]$ and all $(x, y) \in \mathbb{R}^{N} \times \mathbb{R}^{N}$ with $|x|+|y| \geq M_{1}$. 
(K2) There exists a positive constant $M_{2}$ such that

$$
\begin{aligned}
& \left(\nabla_{x} K(t, x, y), x\right)+\left(\nabla_{y} K(t, x, y), y\right) \\
& \quad \leq \max \{p, q\} K(t, x, y)
\end{aligned}
$$

for a.e. $t \in[0, T]$ and all $(x, y) \in \mathbb{R}^{N} \times \mathbb{R}^{N}$ with $|x|+|y| \geq M_{2}$.

(K3) There exists positive constants $c_{1}, c_{2}, M_{3}$ such that

$$
K(t, x, y) \geq c_{1}|x|^{p}+c_{2}|y|^{q}
$$

for a.e. $t \in[0, T]$ and all $(x, y) \in \mathbb{R}^{N} \times \mathbb{R}^{N}$ with $|x|+|y| \leq M_{3}$.

(F1) There exist $L_{1} \in(0,1], b_{1} \in\left(0, \min \left\{\rho^{-} / p, c_{1}\right\}\right), b_{2} \in$ $\left(0, \min \left\{\gamma^{-} / q, c_{2}\right\}\right)$ such that

$$
F(t, x, y) \leq b_{1}|x|^{p}+b_{2}|y|^{q}
$$

for a.e. $t \in[0, T]$ and all $(x, y) \in \mathbb{R}^{N} \times \mathbb{R}^{N}$ with $|x|+|y| \leq L_{1}$.

$(F 2)$

$$
\lim _{|x|+|y| \rightarrow+\infty} \frac{F(t, x, y)}{|x|^{p}+|y|^{q}}=+\infty
$$

uniformly for a.e. $t \in[0, T]$.

(F3) There exist constants $\xi, \eta_{1}, \eta_{2}, L_{2} \in(0,+\infty)$ and $v_{1}, v_{2} \in[0, \min \{p, q\})$ such that

$$
\begin{gathered}
\left(\max \{p, q\}+\frac{1}{\xi+\eta_{1}|x|^{\nu_{1}}+\eta_{2}|y|^{\nu_{2}}}\right) F(t, x, y) \\
\leq\left(\nabla_{x} F(t, x, y), x\right)+\left(\nabla_{y} F(t, x, y), y\right)
\end{gathered}
$$

for a.e. $t \in[0, T]$ and all $(x, y) \in \mathbb{R}^{N} \times \mathbb{R}^{N}$ with $|x|+|y|>L_{2}$.

(I1) There exist constants $d_{3}>0, d_{4}>0, \theta_{3} \in$ $[0, p), G_{1}>0$ such that

$$
\begin{aligned}
d_{3}|x|^{p} \geq I_{i}(x) \geq-d_{4}|x|^{\theta_{3}}, & \left(\nabla I_{i}(x), x\right) \leq \max \{p, q\} I_{i}(x)
\end{aligned}
$$

for all $x \in \mathbb{R}^{N}$ with $|x| \geq G_{1}, i=1, \ldots, l$.

(H1) There exist constants $d_{5}>0, d_{6}>0, \theta_{4} \in[0, q)$, $G_{2}>0$ such that

$$
\begin{aligned}
d_{5}|y|^{q} \geq H_{j}(y) \geq & -d_{6}|y|^{\theta_{4}}, \\
& \left(\nabla H_{j}(y), y\right) \leq \max \{p, q\} H_{j}(y)
\end{aligned}
$$

for all $y \in \mathbb{R}^{N}$ with $|y| \geq G_{2}, j=1, \ldots, m$.

(I2) There exist constants $d_{7}>0, \theta_{5} \in[0, \min \{p, q\})$, $G_{3}>0$ such that

$$
I_{i}(x) \geq-d_{7}|x|^{\theta_{5}}
$$

for all $x \in \mathbb{R}^{N}$ with $|x| \leq G_{3}, i=1, \ldots, l$.

(H2) There exist constants $d_{8}>0, \theta_{6} \in[0, \min \{p, q\})$, $G_{4}>0$ such that

$$
H_{j}(y) \geq-d_{8}|y|^{\theta_{6}}
$$

for all $y \in \mathbb{R}^{N}$ with $|y| \leq G_{4}, j=1, \ldots, m$.
(I3) $I_{i}(0)=0, I_{i}(x)$ is even in $x \in \mathbb{R}^{N}, i=1, \ldots, l$.

(H3) $H_{j}(0)=0, H_{j}(y)$ is even in $y \in \mathbb{R}^{N}, j=1, \ldots, m$.

Then system (1) has an unbounded sequence of weak solutions.

Theorem 2. Suppose that (W0), (A), (W1), (K1)-(K3), (F1)-(F3), (I1), (H1), (I3), (H3), and the following conditions hold.

$(\mathrm{IH} 2)^{\prime}$ There exist positive constants $d_{7}^{\prime}, d_{8}^{\prime}$ with

$$
\begin{aligned}
& d_{7}^{\prime} l C_{\alpha, p, \infty}^{\min \{p, q\}}+d_{8}^{\prime} m C_{\beta, q, \infty}^{\min \{p, q\}} \\
& \leq \min \left\{\min \left\{\frac{\rho^{-}}{p}, c_{1}\right\}-b_{1}, \min \left\{\frac{\gamma^{-}}{q}, c_{2}\right\}-b_{2}\right\} \\
& \cdot \frac{1}{\max \left\{2^{q-1}, 2^{p-1}\right\}},
\end{aligned}
$$

and $G_{3}^{\prime}>0$ such that

$$
I_{i}(x) \geq-d_{7}^{\prime}|x|^{\min \{p, q\}}
$$

for all $x \in \mathbb{R}^{N}$ with $|x| \leq G_{3}^{\prime}, i=1, \ldots, l$, and $G_{4}^{\prime}>0$ such that

$$
H_{j}(y) \geq-d_{8}^{\prime}|y|^{\min \{p, q\}}
$$

for all $y \in \mathbb{R}^{N}$ with $|y| \leq G_{4}^{\prime}, j=1, \ldots, m$, where

$$
\begin{array}{ll}
C_{\alpha, p, \infty}=\frac{T^{\alpha-1 / p}}{\Gamma(\alpha)\left(\alpha p-p^{\prime}+1\right)^{1 / p^{\prime}}}, & p^{\prime}=\frac{p}{p-1}, \\
C_{\beta, q, \infty}=\frac{T^{\beta-1 / q}}{\Gamma(\beta)\left(\beta q-q^{\prime}+1\right)^{1 / q^{\prime}}}, & q^{\prime}=\frac{q}{q-1} .
\end{array}
$$

Then system (1) has an unbounded sequence of weak solutions.

It is easy to prove that the following $(\mathrm{AR})^{\prime}$ condition implies that (F2) and (F3) hold.

$(\mathrm{AR})^{\prime}$ there are constants $\mu>\max \{p, q\}, L_{2}>0$ such that

$$
\begin{aligned}
0 & <\mu F(t, x, y) \\
& \leq\left(\nabla_{x} F(t, x, y), x\right)+\left(\nabla_{y} F(t, x, y), y\right)
\end{aligned}
$$

for a.e. $t \in[0, T]$ and all $(x, y) \in \mathbb{R}^{N} \times \mathbb{R}^{N}$ with $|x|+|y| \geq L_{2}$. Indeed, obviously, (AR) ${ }^{\prime}$ implies that $(F 3)$ holds with $\nu_{1}=$ $\nu_{2}=0$ and $1 / \xi=\mu-\max \{p, q\}$. Moreover, by the proof of Theorem 1.2 in [4], (AR) ${ }^{\prime}$ and (W0) imply that there exist positive constants $B_{i}, i=1,2,3,4$ such that

$$
F(t, x, y) \geq B_{1}|x|^{\mu}+B_{2}|y|^{\mu}-B_{3}-B_{4} b(t)
$$

for all $(x, y) \in \mathbb{R}^{N} \times \mathbb{R}^{N}$ and a.e. $t \in[0, T]$, and so it is easy to see that (F2) holds. Then by Theorems 1 and 2, we have the following corollaries.

Corollary 3. Suppose that (W0), (A), (W1), (K1)-(K3), (F1), (I1)-(I3), (H1)-(H3), and (AR)' hold. Then system (1) has an unbounded sequence of weak solutions. 
Corollary 4. Assume that (W0), (A), (W1), (K1)-(K3), (F1), (I1), (I3), (H1), (H3), (IH2)', and $(A R)^{\prime}$ hold. Then system (1) has an unbounded sequence of weak solutions.

Remark 5. There exist examples satisfying Theorems 1 and 2. For example, let $p>1, q>1, \rho(t)=\gamma(t)=t^{2}+1, I_{i}(x)=$ $|x|^{p}, i=1, \ldots, l, H_{j}(y)=|y|^{p}, j=1, \ldots, m$, and

$$
\begin{aligned}
& F(t, x, y)=\left(t^{2}+1\right)\left(|x|^{\max \{p, q\}}+|y|^{\max \{p, q\}}\right) \\
& \cdot \ln \left(1+|x|^{p}+|y|^{q}\right), \\
& K(t, x, y)=\frac{t^{2}+1}{\theta_{1}}|x|^{\theta_{1}}+\frac{t^{2}+1}{\theta_{2}}|y|^{\theta_{2}}
\end{aligned}
$$

for all $(x, y) \in \mathbb{R}^{N} \times \mathbb{R}^{N}$ and a.e. $t \in[0, T]$, where $\theta_{1} \in(0, p]$ and $\theta_{2} \in(0, q]$.

With similar proofs of Theorems 1 and 2, we can obtain the corresponding theorems for the following $p$-Laplacian system:

$$
\begin{gathered}
{ }_{t} D_{T}^{\alpha}\left(\rho(t) \Phi_{p}\left({ }_{0}^{c} D_{t}^{\alpha} u(t)\right)\right)=\nabla_{u} W(t, u(t)), \\
\text { a.e. } t \in[0, T], \\
\Delta\left({ }_{t} D_{T}^{\alpha-1}\left(\rho \Phi_{p}\left({ }_{0}^{c} D_{t}^{\alpha} u\right)\right)\right)\left(t_{i}\right)=\nabla I_{i}\left(u\left(t_{i}\right)\right), \\
i=1,2, \ldots, l, \\
u(0)=u(T)=0,
\end{gathered}
$$

where $W(t, x)=-K(t, x)+F(t, x)$ for all $x \in \mathbb{R}^{N}(N \geq 1)$ and a.e. $t \in[0, T]$.

Theorem 6. Suppose that the following conditions hold.

$(W 0)^{\prime} W(t, x)$ is measurable in $t$ for each $x \in \mathbb{R}^{N}$, continuously differential in $x \in \mathbb{R}^{N}$ for a.e. $t \in[0, T]$, and there exist $a \in C\left(\mathbb{R}^{+}, \mathbb{R}^{+}\right)$and $b \in L^{1}\left([0, T] ; \mathbb{R}^{+}\right)$such that

$$
\begin{aligned}
& |K(t, x)|+|\nabla K(t, x)|+|F(t, x)|+|\nabla F(t, x)| \\
& \quad \leq a(|x|) b(t), \\
& \left|I_{i}(x)\right|+\left|\nabla I_{i}(x)\right| \leq a(|x|), \quad i=1,2, \ldots, l
\end{aligned}
$$

for all $x \in \mathbb{R}^{N}$ and a.e. $t \in[0, T]$.

$(\mathscr{A})^{\prime} \rho^{-}:=\operatorname{essinf}_{[0, T]} \rho(t)>0$.

$(W 1)^{\prime} W(t, x)$ is even in $x \in \mathbb{R}^{N}$ and $W(t, 0) \equiv 0$ for a.e. $t \in[0, T]$.

$(K 1)^{\prime}$ There exist $\theta_{1} \in[0, p), d_{1}>0, M_{1}>0$ such that

$$
K(t, x) \geq-d_{1}|x|^{\theta_{1}}
$$

for a.e. $t \in[0, T]$ and all $x \in \mathbb{R}^{N}$ with $|x| \geq M_{1}$.

$(K 2)^{\prime}$ There exists a positive constant $M_{2}$ such that

$$
\left(\nabla_{x} K(t, x), x\right) \leq p K(t, x),
$$

for a.e. $t \in[0, T]$ and all $x \in \mathbb{R}^{N}$ with $|x| \geq M_{2}$.
$(K 3)^{\prime}$ There exist positive constants $c, M_{3}$ such that

$$
K(t, x) \geq c|x|^{p}
$$

for a.e. $t \in[0, T]$ and all $x \in \mathbb{R}^{N}$ with $|x| \leq M_{3}$. that

$(F 1)^{\prime}$ There exist $L_{1} \in(0,1], b \in\left(0, \min \left\{\rho^{-} / p, c\right\}\right)$ such

$$
F(t, x) \leq b|x|^{p}
$$

for a.e. $t \in[0, T]$ and all $x \in \mathbb{R}^{N}$ with $|x| \leq L_{1}$.

$(F 2)^{\prime}$

$\lim _{|x| \rightarrow+\infty} \frac{F(t, x)}{|x|^{p}}=+\infty \quad$ uniformly for a.e. $t \in[0, T]$.

$(F 3)^{\prime}$ There exist constants $\xi, \eta, L_{2} \in(0,+\infty)$ and $\nu \in$ $[0, p)$ such that

$$
\left(p+\frac{1}{\xi+\eta|x|^{\nu}}\right) F(t, x, y) \leq\left(\nabla_{x} F(t, x), x\right)
$$

for a.e. $t \in[0, T]$ and all $x \in \mathbb{R}^{N}$ with $|x|>L_{2}$.

$(I 1)^{\prime}$ There exist constants $d_{2}>0, \theta_{2} \in[0, p), G_{1}>0$ such that

$$
I_{i}(x) \geq-d_{2}|x|^{\theta_{2}}, \quad\left(\nabla I_{i}(x), x\right) \leq p I_{i}(x)
$$

for all $x \in \mathbb{R}^{N}$ with $|x| \geq G_{1}, i=1, \ldots, l$.

$(I 2)^{\prime}$ There exist constants $d_{3}>0, \theta_{3} \in[0, p), G_{2}>0$ such that

$$
I_{i}(x) \geq-d_{3}|x|^{\theta_{3}}
$$

for all $x \in \mathbb{R}^{N}$ with $|x| \leq G_{2}, i=1, \ldots, l$.

$(I 3)^{\prime} I_{i}(0)=0, I_{i}(x)$ is even in $x \in \mathbb{R}^{N}, i=1, \ldots, l$.

Then system (29) has an unbounded sequence of weak solutions.

Theorem 7. Suppose that $(W 0)^{\prime},(\mathscr{A})^{\prime},(W 1)^{\prime},(K 1)^{\prime}-(K 3)^{\prime}$, $(F 1)^{\prime}-(F 3)^{\prime},(I 1)^{\prime},(I 3)^{\prime}$, and the following condition holds.

$(I 2)^{\prime \prime}$ There exist positive constants $d_{2}^{\prime}$ with

$$
d_{2}^{\prime} l C_{\alpha, p, \infty}^{p} \leq \min \left\{\frac{\rho^{-}}{p}, c_{1}\right\}-b_{1},
$$

and $G_{2}^{\prime}>0$ such that

$$
I_{i}(x) \geq-d_{2}^{\prime}|x|^{p}
$$

for all $x \in \mathbb{R}^{N}$ with $|x| \leq G_{2}^{\prime}, i=1, \ldots, l$.

Then system (29) has an unbounded sequence of weak solutions.

Corollary 8. Suppose that $(W 0)^{\prime},(\mathscr{A})^{\prime},(W 1)^{\prime},(K 1)^{\prime}-(K 3)^{\prime}$, $(F 1)^{\prime}$, and $(I 1)^{\prime}-(I 3)^{\prime}$ and the following condition holds.

$(A R)^{\prime \prime}$ There are constants $\mu>p, L_{2}>0$ such that

$$
0<\mu F(t, x) \leq\left(\nabla_{x} F(t, x), x\right)
$$

for a.e. $t \in[0, T]$ and all $x \in \mathbb{R}^{N}$ with $|x| \geq L_{2}$.

Then system (29) has an unbounded sequence of weak solutions. 
Corollary 9. Assume that $(W 0)^{\prime},(\mathscr{A})^{\prime},(W 1)^{\prime},(K 1)^{\prime}-(K 3)^{\prime}$, $(F 1)^{\prime},(I 1)^{\prime},(I 2)^{\prime \prime},(I 3)^{\prime}$, and $(A R)^{\prime \prime}$ hold. Then system (29) has an unbounded sequence of weak solutions.

Remark 10. Corollaries 8 and 9 are still different from Theorem B. Indeed, if $N=1, \rho(t) \equiv 1$, and $K(t, x) \equiv$ $(1 / p)|x|^{p}$ for a.e. $t \in[0, T]$ and all $x \in \mathbb{R}$, system (29) reduces to (9). However, it is easy to see that $(I 1)^{\prime}$ and $(I 2)^{\prime}\left(\right.$ or $\left.(I 2)^{\prime \prime}\right)$ are different from $(A 1)$. There exist examples satisfying $(I 1)^{\prime}$ and $(I 2)^{\prime}$ but not satisfying $(A 1)$ and $(A 4)$. For example, let $N=1$ and $I_{i}(x)=|x|^{p}$ for all $x \in \mathbb{R}, i=1, \ldots, l$. Then $I_{i}^{\prime}(x)=p|x|^{p-2} x$. It is easy to see that $I_{i}, i=1, \ldots, l$ satisfy $(I 1)^{\prime}$ and $(I 2)^{\prime}$. Set $Q_{i}(x)=I_{i}^{\prime}(x), i=1, \ldots, l$. Obviously, $Q_{i}(x), i=1, \ldots, l$ do not satisfy $(A 1)$. Moreover, there exist examples satisfying $(F 1)^{\prime}-(F 3)^{\prime}$ but not satisfying $(A 2)$. For example, let

$$
F(t, x) \equiv F(x)=|x|^{p} \ln \left(1+|x|^{p}\right)
$$

for a.e. $t \in[0, T]$. Finally, one can also establish some results which are similar to Theorem A for system (1) and system (29) by combining those assumptions and arguments of Theorems 1 and 2 with those ideas proving Theorem A.

\section{Preliminaries}

In this section, we recall some known definitions and lemmas about fractional derivatives. For more details, the readers can see $[12,39-42]$.

Let $a, b \in(-\infty,+\infty)$ and

$$
A C([a, b]):=\{u:[a, b]
$$

$\longrightarrow \mathbb{R}^{N} \mid u$ is absolutely continuous on $\left.[a, b]\right\}$.

Definition 11 (see $[40,42]$ ). Let $f \in A C[a, b]$ and $\varrho \in(0,1)$. ${ }_{a} D_{t}^{\varrho}$ and ${ }_{t} D_{b}^{\varrho}$ denote the left and right Riemann-Liouville fractional derivatives of order $\alpha$ for function $f$, respectively, which are defined by

$$
\begin{aligned}
& { }_{a} D_{t}^{\varrho} f(t)=\frac{1}{\Gamma(1-\varrho)} \frac{d}{d t} \int_{a}^{t}(t-s)^{-\varrho} f(s) d s, \quad t>a, \\
& { }_{t} D_{b}^{\varrho} f(t)=-\frac{1}{\Gamma(1-\varrho)} \frac{d}{d t} \int_{a}^{t}(s-t)^{-\varrho} f(s) d s,
\end{aligned}
$$

$$
t<b \text {. }
$$

Definition 12 (see $[40,42])$. Let $f \in A C[a, b]$ and $\varrho \in(0,1)$. ${ }_{a}^{c} D_{t}^{\varrho}$ and ${ }_{t}^{c} D_{b}^{\varrho}$ denote the left and right Caputo fractional derivatives of order $\varrho$ for function $f$, respectively, which are defined by

$$
\begin{aligned}
{ }_{a}^{c} D_{t}^{\varrho} f(t) & ={ }_{a} D_{t}^{\varrho-1} f^{\prime}(t) \\
& =\frac{1}{\Gamma(1-\varrho)} \int_{a}^{t}(t-s)^{-\varrho} f^{\prime}(s) d s, \\
{ }_{t}^{c} D_{b}^{\varrho} f(t) & ={ }_{t} D_{b}^{\varrho-1} f^{\prime}(t) \\
& =\frac{1}{\Gamma(1-\varrho)} \int_{t}^{b}(s-t)^{-\varrho} f^{\prime}(s) d s .
\end{aligned}
$$

Remark 13 (see $[40,42])$. When $\varrho=1,{ }_{a} D_{t}^{\varrho} f(t)={ }_{a}^{c} D_{t}^{\varrho} f(t)=$ $f^{\prime}(t)$ and ${ }_{t} D_{b}^{\varrho} f(t)={ }_{t}^{c} D_{b}^{\varrho} f(t)=-f^{\prime}(t)$.

$$
\begin{aligned}
& \text { Let } \\
& C_{0}^{\infty}\left([0, T], \mathbb{R}^{N}\right) \\
& \quad:=\left\{u \mid u \in C^{\infty}\left([0, T], \mathbb{R}^{N}\right), u(0)=u(T)=0\right\}
\end{aligned}
$$

with the norm $\|u\|_{\infty}=\max _{[0, T]}|u(t)|$, and, for $s>1$,

$$
\begin{aligned}
& L^{s}\left([0, T], \mathbb{R}^{N}\right) \\
& \quad:=\left\{\left.u\left|u:[0, T] \longrightarrow \mathbb{R}^{N}, \int_{0}^{T}\right| u(t)\right|^{s} d t<\infty\right\}
\end{aligned}
$$

with the norm $\|u\|_{L^{s}}=\left(\int_{0}^{T}|u(t)|^{s} d t\right)^{1 / s}$.

For $\varrho \in(0,1]$ and $s>1$, we define $E_{0}^{\varrho, s}(0, T)$ as the closure of $C_{0}^{\infty}\left([0, T], \mathbb{R}^{N}\right)$, with respect to the norm:

$$
\|u\|_{s}=\left(\int_{0}^{T}\left|{ }_{0}^{c} D_{t}^{\varrho} u(t)\right|^{s} d t+\int_{0}^{T}|u(t)|^{s} d t\right)^{1 / s}
$$

$$
\forall u \in E_{0}^{e, s}(0, T) .
$$

Then by Proposition 3.1 in [12], $E$ is separable and reflexive Banach space, and if $u \in E_{0}^{\rho, s}(0, T)$, then $u,{ }_{0}^{c} D_{t}^{\varrho} u \in$ $L^{s}([0, T], \mathbb{R})$ and $u(0)=u(T)=0$. Moreover, by Remark 3.1 in $[12],{ }_{0}^{c} D_{t}^{Q} u(t)={ }_{0} D_{t}^{Q} u(t), t \in[0, T]$.

Proposition 14 (see [12]). Assume that $\varrho \in(0,1]$ and $s>1$. For all $u \in E_{0}^{\varrho, s}(0, T)$,

$$
\|u\|_{L^{s}} \leq C_{\varrho}\left\|_{0}^{c} D_{t}^{\varrho} u\right\|_{L^{s}},
$$

where $C_{\varrho}=T^{\varrho} / \Gamma(\varrho+1)$. Moreover, if $\varrho>1 / s$, then

$$
\|u\|_{\infty} \leq C_{\varrho, s, \infty}\left\|{ }_{0}^{c} D_{t}^{Q} u\right\|_{L^{s}},
$$

where $C_{\varrho, s, \infty}:=T^{\varrho-1 / s} / \Gamma(\varrho)\left(\varrho s-s^{\prime}+1\right)^{1 / s^{\prime}}$ and $s^{\prime}=s /(s-1)$.

By Proposition 14, it is easy to obtain that

$$
\|u\|_{\infty} \leq C_{\varrho, s, \infty}\left\|{ }_{0}^{c} D_{t}^{\varrho} u\right\|_{L^{s}} \leq C_{\varrho, s, \infty}\|u\|_{s} .
$$

Proposition 15 (see [12]). Assume that $1 / p<\alpha \leq 1$ and $1<$ $p<\infty$, and the sequence $\left\{u_{k}\right\}$ converges weakly to $u$ in $E_{0}^{\alpha, p}$. Then $u_{k} \rightarrow u$ in $C\left([0, T], \mathbb{R}^{N}\right)$.

Assume that $\alpha, \beta \in(0,1]$. Let $E=E_{0}^{\alpha, p}(0, T) \times E_{0}^{\beta, q}(0, T)$. On $E$, define the norm:

$$
\|(u, v)\|_{E}=\|u\|_{p}+\|v\|_{q}
$$

for all $(u, v) \in E_{0}^{\alpha, p}(0, T) \times E_{0}^{\beta, q}(0, T)=E$.

Similar to Definitions 2.4 and 2.5 in [35], we also present the following two definitions. 
Definition 16. Let

$$
\begin{gathered}
(u, v) \in\{(u, v) \in A C([0, T]) \times A C([0, T]): \\
\int_{t_{i}}^{t_{i+1}}\left(|u(t)|^{p}+\left|{ }_{0}^{c} D_{t}^{\alpha} u(t)\right|^{p}\right) d t<\infty, \\
\quad \int_{s_{j}}^{s_{j+1}}\left(|v(t)|^{q}+\left|{ }_{0}^{c} D_{t}^{\alpha} u(t)\right|^{q}\right) d t<\infty, i \\
=0,1, \ldots, l, j=0,1, \ldots, m\} .
\end{gathered}
$$

If $(u, v)$ satisfies the first equation of (1) for a.e. $t \in[0, T] \backslash$ $\left\{t_{1}, \ldots, t_{l}\right\}$ and the second equation of (1) for a.e. $t \in[0, T] \backslash$ $\left\{s_{1}, \ldots, s_{m}\right\}, \quad{ }_{t} D_{T}^{\alpha-1} \Phi_{p}\left({ }_{0}^{c} D_{t}^{\alpha} u\right)\left(t_{i}^{+}\right), \quad{ }_{t} D_{T}^{\alpha-1} \Phi_{p}\left({ }_{0}^{c} D_{t}^{\alpha} u\right)\left(t_{i}^{-}\right)$, ${ }_{t} D_{T}^{\beta-1} \Phi_{q}\left({ }_{0}^{c} D_{t}^{\beta} v\right)\left(s_{j}^{+}\right)$, and ${ }_{t} D_{T}^{\alpha-1} \Phi_{p}\left({ }_{0}^{c} D_{t}^{\beta} v\right)\left(s_{j}^{-}\right)$exist and satisfy the impulsive conditions of (1), and boundary conditions $u(0)=u(T)=0$ and $v(0)=v(T)=0$, then we call $(u, v)$ a classical solution of (1).

Definition 17. For any $(h, w) \in E$, if the following two equalities

$$
\begin{gathered}
\int_{0}^{T}\left(\rho(t)\left|{ }_{0}^{c} D_{t}^{\alpha} u(t)\right|^{p-2}{ }_{0}^{c} D_{t}^{\alpha} u(t),{ }_{0}^{c} D_{t}^{\alpha} h(t)\right) d t \\
+\sum_{i=1}^{l}\left(\nabla I_{i}\left(u\left(t_{i}\right)\right), h\left(t_{i}\right)\right) \\
\quad-\int_{0}^{T}\left(\nabla_{u} W(t, u(t), v(t)), h(t)\right) d t=0, \\
\int_{0}^{T}\left(\gamma(t)\left|{ }_{0}^{c} D_{t}^{\beta} v(t)\right|^{q-2}{ }_{0}^{c} D_{t}^{\beta} v(t),{ }_{0}^{c} D_{t}^{\beta} h(t)\right) d t \\
+\sum_{j=1}^{m}\left(H_{j}\left(u\left(t_{i}\right)\right), w\left(s_{j}\right)\right) \\
-\int_{0}^{T}\left(\nabla_{v} W(t, u(t), v(t)), h(t)\right) d t=0
\end{gathered}
$$

hold then the vector function $(u, v) \in E$ is called a weak solution of (1).

For $(u, v) \in E$, we define the functional $\mathscr{J}: E \rightarrow \mathbb{R}$ by

$$
\begin{aligned}
\mathscr{J}(u, v)= & \frac{1}{p} \int_{0}^{T} \rho(t)\left|{ }_{0}^{c} D_{t}^{\alpha} u(t)\right|^{p} d t \\
& +\frac{1}{q} \int_{0}^{T} \gamma(t)\left|{ }_{0}^{c} D_{t}^{\beta} v(t)\right|^{q} d t+\sum_{i=1}^{l} I_{i}\left(u\left(t_{j}\right)\right) \\
& +\sum_{j=1}^{m} H_{j}\left(v\left(s_{j}\right)\right)+\int_{0}^{T} K(t, u(t), v(t)) d t \\
& -\int_{0}^{T} F(t, u(t), v(t)) d t \\
= & \Phi(u, v)+\Psi(u, v)
\end{aligned}
$$

where

$$
\begin{aligned}
\Phi(u, v)= & \frac{1}{p} \int_{0}^{T} \rho(t)\left|{ }_{0}^{c} D_{t}^{\alpha} u(t)\right|^{p} d t \\
& +\frac{1}{q} \int_{0}^{T} \gamma(t)\left|{ }_{0}^{c} D_{t}^{\beta} v(t)\right|^{q} d t \\
& +\int_{0}^{T} K(t, u(t), v(t)) d t \\
& -\int_{0}^{T} F(t, u(t), v(t)) d t, \\
\Psi(u, v)= & \sum_{i=1}^{l} I_{i}\left(u\left(t_{i}\right)\right)+\sum_{j=1}^{m} H_{j}\left(v\left(s_{j}\right)\right) .
\end{aligned}
$$

It follows from (W0), the continuity of $\nabla I_{i}$ and $\nabla H_{j}$ and Theorem 5.41 in [42], that $\Phi$ and $\Psi$ are continuously differentiable and so $\mathscr{J} \in C^{1}(E, \mathbb{R})$ and

$$
\begin{aligned}
\left\langle\mathcal{F}^{\prime}\right. & (u, v),(h, w)\rangle \\
= & \int_{0}^{T}\left(\rho(t)\left|{ }_{0}^{c} D_{t}^{\alpha} u(t)\right|_{0}^{p-2} D_{t}^{\alpha} u(t),{ }_{0}^{c} D_{t}^{\alpha} h(t)\right) d t \\
& +\int_{0}^{T}\left(\gamma(t)\left|{ }_{0}^{c} D_{t}^{\alpha} v(t)\right|_{0}^{q-2} D_{t}^{\beta} v(t),{ }_{0}^{c} D_{t}^{\alpha} w(t)\right) d t \\
& +\int_{0}^{T}\left(\nabla_{u} K(t, u(t), v(t)), h(t)\right) d t \\
& -\int_{0}^{T}\left(\nabla_{v} F(t, u(t), v(t)), w(t)\right) d t \\
& +\sum_{i=1}^{l}\left(\nabla I_{i}\left(u\left(t_{i}\right)\right), h\left(t_{i}\right)\right) \\
& +\sum_{j=1}^{m}\left(\nabla H_{j}\left(v\left(s_{j}\right)\right), w\left(s_{j}\right)\right) .
\end{aligned}
$$

Hence, the critical point of $\mathscr{J}$ is a weak solution of (1). Similar to the arguments of Propositions 2.5 and 2.6 in [35], it is easy to obtain that $(u, v)$ is a classical solution of $(1)$ if $(u, v) \in E$ is a weak solution of (1).

Assume that $E$ is a real Banach space and $\varphi \in C^{1}(E, \mathbb{R})$. For any sequence $\left\{u_{k}\right\} \subset E$, if $\varphi\left(u_{k}\right)$ is bounded and $\varphi^{\prime}\left(u_{k}\right) \rightarrow$ 0 as $k \rightarrow \infty$, then we call $\left\{u_{k}\right\}$ a Palais-Smale sequence. If any Palais-Smale sequence $\left\{u_{k}\right\}$ has a convergent subsequence, then we call $\varphi$ which satisfies Palais-Smale condition.

Similar to the proofs in [39], we will also use the following symmetric mountain pass theorem to prove our main results.

Lemma 18 (see [2]). Let $E$ be an infinite dimensional Banach space and let $\varphi \in C^{1}(E, \mathbb{R})$ be even and satisfy Palais-Smale condition, and $\varphi(0)=0$. If $E=V \oplus X$, where $V$ is finite dimensional, and $\varphi$ satisfies the following, then $\varphi$ possesses an unbounded sequence of critical values.

(i) There are constants $\rho, \xi>0$ such that $\left.\varphi\right|_{\partial B_{\rho} \cap X} \geq \xi$.

(ii) For each finite dimensional subspace $\widetilde{E} \subset E$, there is $R=R(\widetilde{E})$ such that $\varphi \leq 0$ on $\widetilde{E} \backslash B_{R(\widetilde{E})}$. 
Remark 19. As shown in [43], a deformation lemma can be proved with replacing Palais-Smale condition with Cerami condition, which implies that Lemma 2.1 in [2] is true under Cerami condition. We say that $\varphi$ satisfies Cerami condition; that is, for every sequence $\left\{u_{k}\right\} \subset E,\left\{u_{k}\right\}$ has a convergent subsequence if $\varphi\left(u_{k}\right)$ is bounded and $\left(1+\left\|u_{k}\right\|_{E}\right)\left\|\varphi^{\prime}\left(u_{k}\right)\right\|_{E^{*}} \rightarrow$ 0 as $k \rightarrow \infty$, where $E^{*}$ with the norm $\|\cdot\|_{E^{*}}$ is the dual space of $E$.

\section{Proofs of Theorems}

Lemma 20. Assume that (A), (K1), (K2), (F2), (F3), (I1), and (H1) hold. Then $\mathscr{J}$ satisfies Cerami condition.

Proof. For any sequence $\left\{\left(u_{n}, v_{n}\right)\right\}_{n=1}^{\infty} \subset E$, suppose that there is a positive constant $C_{1}>0$ such that

$$
\begin{aligned}
\left|\mathscr{J}\left(u_{n}, v_{n}\right)\right| & \leq C_{1}, \\
\left(1+\left\|\left(u_{n}, v_{n}\right)\right\|_{E}\right)\left\|\mathscr{J}^{\prime}\left(u_{n}, v_{n}\right)\right\|_{E^{*}} & \leq C_{1} .
\end{aligned}
$$

$\forall n \in \mathbb{N}$

By (F2) and assumption ( $W 0)$, there exist positive constants $A, C_{2}$, and $C_{3}$ such that

$$
\begin{aligned}
& F(t, x, y) \geq A|x|^{p}+A|y|^{q}-C_{2}-C_{3} b(t), \\
& \forall(x, y) \in \mathbb{R}^{N} \times \mathbb{R}^{N} \text {, a.e. } t \in[0, T] .
\end{aligned}
$$

It follows from $(F 3)$ that

$$
\begin{aligned}
& {\left[\left(\nabla_{x} F(t, x, y), x\right)+\left(\nabla_{y} F(t, x, y), y\right)\right.} \\
& \quad-\max \{p, q\} F(t, x, y)]\left(\xi+\eta_{1}|x|^{\nu_{1}}+\eta_{2}|y|^{\nu_{2}}\right) \\
& \quad \geq F(t, x, y)
\end{aligned}
$$

for all $(x, y) \in \mathbb{R}^{N} \times \mathbb{R}^{N}$ with $|x|+|y|>L_{2}$ and a.e. $t \in[0, T]$. Assumption (W0) and (60) imply that there exists a positive constant $C_{4}$ such that

$$
\begin{aligned}
& {\left[\left(\nabla_{x} F(t, x, y), x\right)+\left(\nabla_{y} F(t, x, y), y\right)\right.} \\
& \quad-\max \{p, q\} F(t, x, y)]\left(\xi+\eta_{1}|x|^{\nu_{1}}+\eta_{2}|y|^{\nu_{2}}\right) \\
& \quad \geq F(t, x, y)-C_{4} b(t)
\end{aligned}
$$

for all $(x, y) \in \mathbb{R}^{N} \times \mathbb{R}^{N}$ and a.e. $t \in[0, T]$.
Assume that $p \geq q$. Then, for any $x \in \mathbb{R}^{N}$ with $|x| \geq 1$ and all $y \in \mathbb{R}^{N}$,

$$
\begin{aligned}
(|x|+|y|)^{\min \{p, q\}} & =(|x|+|y|)^{q} \leq 2^{q-1}\left(|x|^{q}+|y|^{q}\right) \\
& \leq 2^{q-1}\left(|x|^{p}+|y|^{q}\right)
\end{aligned}
$$

and, for any $x \in \mathbb{R}^{N}$ with $|x| \leq 1$ and all $y \in \mathbb{R}^{N}$,

$$
\begin{aligned}
(|x|+|y|)^{\min \{p, q\}} & =(|x|+|y|)^{q} \leq 2^{q-1}\left(|x|^{p}+|y|^{q}\right) \\
& \leq 2^{q-1}\left(|x|^{p}+|y|^{q}+1\right) .
\end{aligned}
$$

Then, (62) and (63) imply that

$$
(|x|+|y|)^{\min \{p, q\}} \leq 2^{q-1}\left(|x|^{p}+|y|^{q}+1\right)
$$

for all $(x, y) \in \mathbb{R}^{N} \times \mathbb{R}^{N}$. Similarly, if $q \geq p$, we have

$$
(|x|+|y|)^{\min \{p, q\}} \leq 2^{p-1}\left(|x|^{p}+|y|^{q}+1\right)
$$

for all $(x, y) \in \mathbb{R}^{N} \times \mathbb{R}^{N}$. Combining (64) and (65), we have

$$
|x|^{p}+|y|^{q} \geq \frac{(|x|+|y|)^{\min \{p, q\}}}{\max \left\{2^{q-1}, 2^{p-1}\right\}}-1
$$

for all $(x, y) \in \mathbb{R}^{N} \times \mathbb{R}^{N}$. Moreover, for $\nu_{1}, \nu_{2} \in(1, \infty)$ and $\eta_{1}, \eta_{2} \in(0,+\infty)$, we have

$$
\begin{aligned}
\eta_{1}|x|^{\nu_{1}}+\eta_{2}|y|^{\nu_{2}} & \\
& \leq \max \left\{\eta_{1}, \eta_{2}\right\}\left[(|x|+|y|)^{\nu_{1}}+(|x|+|y|)^{\nu_{2}}\right] \\
& \leq 2 \max \left\{\eta_{1}, \eta_{2}\right\}\left[(|x|+|y|)^{\max \left\{\nu_{1}, \nu_{2}\right\}}+1\right]
\end{aligned}
$$

for all $(x, y) \in \mathbb{R}^{N} \times \mathbb{R}^{N}$, and

$$
\begin{aligned}
\xi+ & 2 \max \left\{\eta_{1}, \eta_{2}\right\} \\
& \leq\left(\xi+2 \max \left\{\eta_{1}, \eta_{2}\right\}\right)(|x|+|y|)^{\max \left\{\nu_{1}, \nu_{2}\right\}} .
\end{aligned}
$$

for all $(x, y) \in \mathbb{R}^{N} \times \mathbb{R}^{N}$ with $|x|+|y| \geq 1$. It follows from assumptions (59), (61), (66), (67), and (68) that there exist positive constants $C_{5}, C_{6}, C_{7}$ such that

$$
\begin{aligned}
& \left(\nabla_{x} F(t, x, y), x\right)+\left(\nabla_{y} F(t, x, y), y\right)-\max \{p, q\} F(t, x, y) \geq \frac{F(t, x, y)-C_{4} b(t)}{\xi+\eta_{1}|x|^{\nu_{1}}+\eta_{2}|y|^{\nu_{2}}} \\
& \quad \geq \frac{A|x|^{p}+A|y|^{q}-C_{2}-C_{3} b(t)-C_{4} b(t)}{\xi+\eta_{1}|x|^{\nu_{1}}+\eta_{2}|y|^{\nu_{2}}} \geq \frac{\left(A / \max \left\{2^{q-1}, 2^{p-1}\right\}\right)(|x|+|y|)^{\min \{p, q\}}-A-C_{2}-C_{3} b(t)-C_{4} b(t)}{\xi+2 \max \left\{\eta_{1}, \eta_{2}\right\}(|x|+|y|)^{\max \left\{\nu_{1}, \nu_{2}\right\}}+2 \max \left\{\eta_{1}, \eta_{2}\right\}} \\
& \quad \geq \frac{\left(A / \max \left\{2^{q-1}, 2^{p-1}\right\}\right)(|x|+|y|)^{\min \{p, q\}}}{\left(\xi+4 \max \left\{\eta_{1}, \eta_{2}\right\}\right)(|x|+|y|)^{\max \left\{\nu_{1}, \nu_{2}\right\}}}-\frac{A+C_{2}+C_{3} b(t)+C_{4} b(t)}{\xi+2 \max \left\{\eta_{1}, \eta_{2}\right\}} \geq C_{5}(|x|+|y|)^{\min \{p, q\}-\max \left\{\nu_{1}, \nu_{2}\right\}}-C_{6} b(t) \\
& -C_{7}
\end{aligned}
$$


for all $(x, y) \in \mathbb{R}^{N} \times \mathbb{R}^{N}$ with $|x|+|y| \geq 1$ and a.e. $t \in[0, T]$. By (69) and assumption (W0), there exist positive constants $C_{8}, C_{9}$ such that

$$
\begin{aligned}
\left(\nabla_{x} F\right. & (t, x, y), x)+\left(\nabla_{y} F(t, x, y), y\right) \\
& \quad-\max \{p, q\} F(t, x, y) \\
\geq & C_{5}(|x|+|y|)^{\min \{p, q\}-\max \left\{v_{1}, v_{2}\right\}}-C_{8} b(t)-C_{9}
\end{aligned}
$$

for all $(x, y) \in \mathbb{R}^{N} \times \mathbb{R}^{N}$ and a.e. $t \in[0, T]$. By $(K 1),(K 2)$, and $(W 0)$, there exists positive constant $C_{10}$ such that

$$
\begin{aligned}
& \max \{p, q\} K(t, x, y)-\left(\nabla_{x} K(t, x, y), x\right) \\
& \quad-\left(\nabla_{y} K(t, x, y), y\right) \geq-C_{10} b(t), \\
& K(t, x, y) \geq-d_{1}|x|^{\theta_{1}}-d_{2}|y|^{\theta_{2}}-C_{10} b(t)-C_{10}
\end{aligned}
$$

for all $(x, y) \in \mathbb{R}^{N} \times \mathbb{R}^{N}$ and a.e. $t \in[0, T]$. Moreover, by $(I 1),(H 1)$, and $(W 0)$, there exist positive constants $C_{11}$ and $C_{12}$ such that

$$
\begin{gathered}
\max \{p, q\} I_{i}(x)-\left(\nabla I_{i}(x), x\right) \geq-C_{11}, \\
I_{i}(x) \geq-d_{3}|x|^{\theta_{3}}-C_{11}, i=1, \ldots, l, \\
\max \{p, q\} H_{j}(y)-\left(\nabla H_{j}(y), y\right) \geq-C_{12}, \\
H_{j}(x) \geq-d_{4}|y|^{\theta_{4}}-C_{12}, j=1, \ldots, m
\end{gathered}
$$

for all $(x, y) \in \mathbb{R}^{N} \times \mathbb{R}^{N}$. By (58) and (70), we have

$$
\begin{aligned}
& (\max \{p, q\}+1) C_{1} \geq \max \{p, q\} \mathscr{J}\left(u_{n}, v_{n}\right) \\
& -\left\langle\mathcal{J}^{\prime}\left(u_{n}, v_{n}\right),\left(u_{n}, v_{n}\right)\right\rangle \\
& \geq \int_{0}^{T}\left[-\left(\nabla_{u} K\left(t, u_{n}(t), v_{n}(t)\right), u_{n}(t)\right)\right. \\
& -\left(\nabla_{v} K\left(t, u_{n}(t), v_{n}(t)\right), v_{n}(t)\right) \\
& \left.+\max \{p, q\} K\left(t, u_{n}(t), v_{n}(t)\right)\right] d t \\
& +\int_{0}^{T}\left[\left(\nabla_{u} F\left(t, u_{n}(t), v_{n}(t)\right), u_{n}(t)\right)\right. \\
& +\left(\nabla_{v} F\left(t, u_{n}(t), v_{n}(t)\right), v_{n}(t)\right) \\
& \left.-\max \{p, q\} F\left(t, u_{n}(t), v_{n}(t)\right)\right] d t \\
& +\sum_{i=1}^{l}\left[\max \{p, q\} I_{i}\left(u_{n}\left(t_{i}\right)\right)\right. \\
& \left.-\left(\nabla I_{i}\left(u_{n}\left(t_{i}\right)\right), u_{n}\left(t_{i}\right)\right)\right] \\
& +\sum_{j=1}^{m}\left[\max \{p, q\} K_{j}\left(v_{n}\left(s_{j}\right)\right)\right. \\
& \left.-\left(\nabla K_{j}\left(v_{n}\left(s_{j}\right)\right), v_{n}\left(s_{j}\right)\right)\right] \\
& \geq \int_{0}^{T}\left[\left(\nabla_{u} F\left(t, u_{n}(t), v_{n}(t)\right), u_{n}(t)\right)\right.
\end{aligned}
$$

$$
\begin{aligned}
& +\left(\nabla_{v} F\left(t, u_{n}(t), v_{n}(t)\right), v_{n}(t)\right) \\
& \left.-\max \{p, q\} F\left(t, u_{n}(t), v_{n}(t)\right)\right] d t \\
& -C_{10} \int_{0}^{T} b(t) d t-C_{11} l-C_{12} m \\
& \geq C_{5} \int_{0}^{T}\left(\left|u_{n}(t)\right|+\left|v_{n}(t)\right|\right)^{\min \{p, q\}-\max \left\{v_{1}, v_{2}\right\}} d t \\
& -\left(C_{8}+C_{10}\right) \int_{0}^{T} b(t) d t-C_{9} T-C_{11} l-C_{12} m,
\end{aligned}
$$

which implies that there exists a positive constant $C_{13}$ such that $\int_{0}^{T}\left(\left|u_{n}(t)\right|+\left|v_{n}(t)\right|\right)^{\min \{p, q\}-\max \left\{v_{1}, v_{2}\right\}} d t<C_{13}$. Then $\int_{0}^{T}\left|u_{n}(t)\right|^{\min \{p, q\}-\max \left\{v_{1}, v_{2}\right\}} d t \quad<\quad C_{13}$ and $\int_{0}^{T}\left|v_{n}(t)\right|^{\min \{p, q\}-\max \left\{v_{1}, v_{2}\right\}} d t<C_{13}$. By (60) and (F2), there exists $C_{14}>0$ such that

$$
\begin{aligned}
& {\left[\left(\nabla_{x} F(t, x, y), x\right)+\left(\nabla_{y} F(t, x, y), y\right)\right.} \\
& \quad-\max \{p, q\} F(t, x, y)]\left(\xi+\eta_{1}|x|^{\nu_{1}}+\eta_{2}|y|^{\nu_{2}}\right) \\
& \quad \geq A|x|^{p}+A|y|^{p}>0
\end{aligned}
$$

for all $(x, y) \in \mathbb{R}^{N} \times \mathbb{R}^{N}$ with $|x|+|y|>L_{2}+C_{14}$ and a.e. $t \in[0, T]$. Let $\widetilde{F}(t, x, y):=\left(\nabla_{x} F(t, x, y), x\right)+\left(\nabla_{y} F(t, x, y), y\right)-$ $\max \{p, q\} F(t, x, y)$ for all $(x, y) \in \mathbb{R}^{N} \times \mathbb{R}^{N}$ and a.e. $t \in[0, T]$, $S_{1}:=\left\{t \in[0, T]|| u_{n}(t)|+| v_{n}(t) \mid>L_{2}+C_{14}\right\}, S_{2}:=[0, T] \backslash S_{1}$, and $\Lambda:=\min \{p, q\}-\max \left\{v_{1}, v_{2}\right\}$. By (58), (W0), (A), (61), (72), (73), (74), (75), and (51), there exist positive constants $C_{15}$ and $C_{16}$ such that

$$
\begin{aligned}
& \frac{\rho^{-}}{p}\left\|u_{n}\right\|_{p}^{p}+\frac{\gamma^{-}}{q}\left\|v_{n}\right\|_{q}^{q} \leq \mathcal{J}\left(u_{n}, v_{n}\right) \\
& \quad-\int_{0}^{T} K\left(t, u_{n}(t), v_{n}(t)\right) d t \\
& \quad+\int_{0}^{T} F\left(t, u_{n}(t), v_{n}(t)\right) d t-\sum_{i=1}^{l} I_{i}\left(u\left(t_{j}\right)\right) \\
& \quad-\sum_{j=1}^{m} H_{j}\left(v\left(s_{j}\right)\right)+\frac{\rho^{-}}{p} \int_{0}^{T}\left|u_{n}(t)\right|^{p} d t+\frac{\gamma^{-}}{q} \\
& \quad \cdot \int_{0}^{T}\left|v_{n}(t)\right|^{q} d t \leq C_{1}+\frac{\rho^{-}}{p} \int_{0}^{T}\left|u_{n}(t)\right|^{p} d t+\frac{\gamma^{-}}{q} \\
& \quad \cdot \int_{0}^{T}\left|v_{n}(t)\right|^{q} d t+d_{1} \int_{0}^{T}\left|u_{n}(t)\right|^{\theta_{1}} d t \\
& \quad+d_{2} \int_{0}^{T}\left|v_{n}(t)\right|^{\theta_{2}} d t+\int_{0}^{T} \widetilde{F}\left(t, u_{n}(t), v_{n}(t)\right) \\
& \quad+\left(\xi+\eta_{1}\left|u_{n}(t)\right|^{\nu_{1}}+\eta_{2}\left|v_{n}(t)\right|^{v_{2}}\right) d t
\end{aligned}
$$


10

Discrete Dynamics in Nature and Society

$$
\begin{aligned}
& +C_{4} \int_{0}^{T} b(t) d t+C_{10} \int_{0}^{T} b(t) d t+C_{10} T \\
& \cdot \int_{0}^{T} b(t) d t+C_{15} \int_{0}^{T} b(t) d t+\left(\xi+\eta_{1}\left\|u_{n}\right\|_{\infty}^{\nu_{1}}\right. \\
& +d_{3} \int_{0}^{T}\left|u_{n}(t)\right|^{\theta_{3}} d t+d_{4} \int_{0}^{T}\left|v_{n}(t)\right|^{\theta_{4}} d t+C_{11} l \\
& \left.+\eta_{2}\left\|v_{n}\right\|_{\infty}^{v_{2}}\right) \int_{0}^{T} \widetilde{F}\left(t, u_{n}(t), v_{n}(t)\right) d t+C_{16}(\xi \\
& +C_{12} m \leq C_{1}+\frac{\rho^{-}}{p} \int_{0}^{T}\left|u_{n}(t)\right|^{p} d t+\frac{\gamma^{-}}{q} \\
& \left.+\eta_{1}\left\|u_{n}\right\|_{\infty}^{\nu_{1}}+\eta_{2}\left\|v_{n}\right\|_{\infty}^{v_{2}}\right) \int_{0}^{T} b(t) d t+C_{10} T+C_{11} l \\
& \cdot \int_{0}^{T}\left|v_{n}(t)\right|^{q} d t+\left(C_{4}+C_{10}\right) \int_{0}^{T} b(t) d t \\
& +C_{12} m+d_{1} T\left\|u_{n}\right\|_{\infty}^{\theta_{1}}+d_{2} T\left\|v_{n}\right\|_{\infty}^{\theta_{2}}+d_{3} T\left\|u_{n}\right\|_{\infty}^{\theta_{3}} \\
& +\int_{S_{2}} \widetilde{F}\left(t, u_{n}(t), v_{n}(t)\right) \\
& +d_{4} T\left\|v_{n}\right\|_{\infty}^{\theta_{4}} \leq C_{1}+\frac{\rho^{-}}{p} \int_{0}^{T}\left|u_{n}(t)\right|^{p} d t+\frac{\gamma^{-}}{q} \\
& \cdot\left(\xi+\eta_{1}\left|u_{n}(t)\right|^{\nu_{1}}+\eta_{2}\left|v_{n}(t)\right|^{\nu_{2}}\right) d t+C_{10} T \\
& \cdot \int_{0}^{T}\left|v_{n}(t)\right|^{q} d t+\left(C_{4}+C_{10}\right) \int_{0}^{T} b(t) d t \\
& +C_{11} l+C_{12} m+\int_{S_{1}} \widetilde{F}\left(t, u_{n}(t), v_{n}(t)\right) \\
& \cdot\left(\xi+\eta_{1}\left|u_{n}(t)\right|^{\nu_{1}}+\eta_{2}\left|v_{n}(t)\right|^{\nu_{2}}\right) d t \\
& +d_{1} \int_{0}^{T}\left|u_{n}(t)\right|^{\theta_{1}} d t+d_{2} \int_{0}^{T}\left|v_{n}(t)\right|^{\theta_{2}} d t \\
& +d_{3} \int_{0}^{T}\left|u_{n}(t)\right|^{\theta_{3}} d t+d_{4} \int_{0}^{T}\left|v_{n}(t)\right|^{\theta_{4}} d t \leq C_{1} \\
& +\frac{\rho^{-}}{p} \int_{0}^{T}\left|u_{n}(t)\right|^{p} d t+\frac{\gamma^{-}}{q} \int_{0}^{T}\left|v_{n}(t)\right|^{q} d t+\left(C_{4}\right. \\
& \left.+C_{10}\right) \int_{0}^{T} b(t) d t+C_{15} \int_{0}^{T} b(t) d t+(\xi \\
& +C_{15} \int_{0}^{T} b(t) d t+\left[(\max \{p, q\}+1) C_{1}\right. \\
& \left.+C_{10} \int_{0}^{T} b(t) d t+C_{11} l+C_{12} m\right]\left(\xi+\eta_{1}\left\|u_{n}\right\|_{\infty}^{\nu_{1}}\right. \\
& \left.+\eta_{2}\left\|v_{n}\right\|_{\infty}^{\nu_{2}}\right)+C_{16}\left(\xi+\eta_{1}\left\|u_{n}\right\|_{\infty}^{v_{1}}+\eta_{2}\left\|v_{n}\right\|_{\infty}^{v_{2}}\right) \\
& \cdot \int_{0}^{T} b(t) d t+C_{10} T+C_{11} l+C_{12} m+d_{1} T\left\|u_{n}\right\|_{\infty}^{\theta_{1}} \\
& +d_{2} T\left\|v_{n}\right\|_{\infty}^{\theta_{2}}+d_{3} T\left\|u_{n}\right\|_{\infty}^{\theta_{3}}+d_{4} T\left\|v_{n}\right\|_{\infty}^{\theta_{4}} \leq C_{1} \\
& +\frac{\rho^{-}}{p}\left\|u_{n}\right\|_{\infty}^{p-\Lambda} \int_{0}^{T}\left|u_{n}(t)\right|^{\Lambda} d t+\frac{\gamma^{-}}{q}\left\|v_{n}\right\|_{\infty}^{q-\Lambda} \\
& \left.+\eta_{1}\left\|u_{n}\right\|_{\infty}^{\nu_{1}}+\eta_{2}\left\|v_{n}\right\|_{\infty}^{\nu_{2}}\right) \int_{S_{1}} \widetilde{F}\left(t, u_{n}(t), v_{n}(t)\right) d t \\
& \cdot \int_{0}^{T}\left|v_{n}(t)\right|^{\Lambda} d t+\left(C_{4}+C_{10}\right) \int_{0}^{T} b(t) d t \\
& +C_{10} T+C_{11} l+C_{12} m+d_{1} T\left\|u_{n}\right\|_{\infty}^{\theta_{1}}+d_{2} T\left\|v_{n}\right\|_{\infty}^{\theta_{2}} \\
& +C_{15} \int_{0}^{T} b(t) d t+\left[(\max \{p, q\}+1) C_{1}\right. \\
& +d_{3} T\left\|u_{n}\right\|_{\infty}^{\theta_{3}}+d_{4} T\left\|v_{n}\right\|_{\infty}^{\theta_{4}} \leq C_{1}+\frac{\rho^{-}}{p} \\
& \left.+C_{10} \int_{0}^{T} b(t) d t+C_{11} l+C_{12} m\right]\left(\xi+\eta_{1}\left\|u_{n}\right\|_{\infty}^{\nu_{1}}\right. \\
& \cdot \int_{0}^{T}\left|u_{n}(t)\right|^{p} d t+\frac{\gamma^{-}}{q} \int_{0}^{T}\left|v_{n}(t)\right|^{q} d t+\left(C_{4}+C_{10}\right) \\
& \left.+\eta_{2}\left\|v_{n}\right\|_{\infty}^{v_{2}}\right)+C_{16}\left(\xi+\eta_{1}\left\|u_{n}\right\|_{\infty}^{v_{1}}+\eta_{2}\left\|v_{n}\right\|_{\infty}^{v_{2}}\right) \\
& \cdot \int_{0}^{T} b(t) d t+C_{15} \int_{0}^{T} b(t) d t+\left(\xi+\eta_{1}\left\|u_{n}\right\|_{\infty}^{\nu_{1}}\right. \\
& \cdot \int_{0}^{T} b(t) d t+C_{10} T+C_{11} l+C_{12} m+d_{1} T\left\|u_{n}\right\|_{\infty}^{\theta_{1}} \\
& \left.+\eta_{2}\left\|v_{n}\right\|_{\infty}^{\nu_{2}}\right) \int_{0}^{T} \widetilde{F}\left(t, u_{n}(t), v_{n}(t)\right) d t-(\xi \\
& +d_{2} T\left\|v_{n}\right\|_{\infty}^{\theta_{2}}+d_{3} T\left\|u_{n}\right\|_{\infty}^{\theta_{3}}+d_{4} T\left\|v_{n}\right\|_{\infty}^{\theta_{4}} \leq C_{1} \\
& +C_{\alpha, p, \infty}^{p-\Lambda} \frac{\rho^{-}}{p}\left\|u_{n}\right\|_{p}^{p-\Lambda} \int_{0}^{T}\left|u_{n}(t)\right|^{\Lambda} d t+C_{\beta, q, \infty}^{q-\Lambda} \\
& \cdot \frac{\gamma^{-}}{q}\left\|v_{n}\right\|_{q}^{q-\Lambda} \int_{0}^{T}\left|v_{n}(t)\right|^{\Lambda} d t+\left(C_{4}+C_{10}\right) \\
& \cdot \int_{0}^{T} b(t) d t+C_{15} \int_{0}^{T} b(t) d t+[(\max \{p, q\}+1) \\
& \cdot \int_{0}^{T}\left|u_{n}(t)\right|^{p} d t+\frac{\gamma^{-}}{q} \int_{0}^{T}\left|v_{n}(t)\right|^{q} d t+\left(C_{4}+C_{10}\right) \\
& \left.\cdot C_{1}+C_{10} \int_{0}^{T} b(t) d t+C_{11} l+C_{12} m\right](\xi
\end{aligned}
$$




$$
\begin{aligned}
& \left.+\eta_{1} C_{\alpha, p, \infty}^{\nu_{1}}\left\|u_{n}\right\|_{p}^{\nu_{1}}+\eta_{2} C_{\beta, q, \infty}^{\nu_{2}}\left\|v_{n}\right\|_{q}^{\nu_{2}}\right)+C_{16}(\xi \\
& \left.+\eta_{1} C_{\alpha, p, \infty}^{v_{1}}\left\|u_{n}\right\|_{p}^{\nu_{1}}+\eta_{2} C_{\beta, q, \infty}^{\nu_{2}}\left\|v_{n}\right\|_{q}^{\nu_{2}}\right) \int_{0}^{T} b(t) d t \\
& +C_{10} T+C_{11} l+C_{12} m+d_{1} T C_{\alpha, p, \infty}^{\theta_{1}}\left\|u_{n}\right\|_{p}^{\theta_{1}} \\
& +d_{2} T C_{\beta, q, \infty}^{\theta_{2}}\left\|v_{n}\right\|_{q}^{\theta_{2}}+d_{3} C_{\alpha, p, \infty}^{\theta_{3}} T\left\|u_{n}\right\|_{p}^{\theta_{3}} \\
& +d_{4} T C_{\beta, q, \infty}^{\theta_{4}}\left\|v_{n}\right\|_{q}^{\theta_{4}} .
\end{aligned}
$$

Since $\max \left\{v_{1}, v_{2}\right\}<\min \{p, q\}, \theta_{1}<p, \theta_{2}<q, \theta_{3}<$ $p, \theta_{4}<q$, and $0<\Lambda<\min \{p, q\}$, the boundness of $\int_{0}^{T}\left|u_{n}(t)\right|^{\Lambda} d t$ and $\int_{0}^{T}\left|v_{n}(t)\right|^{\Lambda} d t$ implies that $\left\|u_{n}\right\|_{p}$ and $\left\|v_{n}\right\|_{q}$ are bounded. Going if necessary to a subsequence, assume that $u_{n} \rightarrow u$ in $E_{0}^{\alpha, p}(0, T)$ and $v_{n} \rightarrow v$ in $E_{0}^{\beta, q}(0, T)$. Then, by Proposition 15, we obtain $\left\|u_{n}-u\right\|_{\infty} \rightarrow 0$ and $\left\|v_{n}-v\right\|_{\infty} \rightarrow$ 0 , and so $\left\|u_{n}-u\right\|_{L^{p}} \rightarrow 0$ and $\left\|v_{n}-v\right\|_{L^{q}} \rightarrow 0$. Similar to the arguments of Lemma 3.1 in [35], we can obtain that $\left\|{ }_{0}^{c} D_{t}^{\alpha} u_{n}(t)-{ }_{0}^{c} D_{t}^{\alpha} u(t)\right\|_{L^{p}} \rightarrow 0$ and $\left\|{ }_{0}^{c} D_{t}^{\alpha} v_{n}(t)-{ }_{0}^{c} D_{t}^{\alpha} v(t)\right\|_{L^{q}} \rightarrow 0$. Therefore, $\left\|u_{n}-u\right\|_{p} \rightarrow 0$ and $\left\|v_{n}-v\right\|_{q} \rightarrow 0$ as $n \rightarrow \infty$, which shows that $\left\|\left(u_{n}, v_{n}\right)-(u, v)\right\|_{E} \rightarrow 0$ as $n \rightarrow \infty$.

Lemma 21. Assume that (A), (K3), (F1), (I2), and (H2) hold. There are constants $\rho, \xi>0$ such that $\left.\mathscr{F}\right|_{\partial B_{\rho} \cap E} \geq \xi$.

Proof. Choosing $\rho=\min \left\{G_{3}, G_{4}, M_{3}, L_{1}\right\} / \max \left\{C_{\alpha, p, \infty}\right.$, $\left.C_{\beta, q, \infty}\right\}$. Then, for all $(u, v) \in E \cap \partial B_{\rho}$, we have $\|u\|_{p}+$ $\|v\|_{q}=\rho$ and then, by (51), $\|u\|_{\infty} \leq C_{\alpha, p, \infty}\|u\|_{p} \leq$ $C_{\alpha, p, \infty} \rho \leq \min \left\{G_{3}, G_{4}, M_{3}, L_{1}\right\}$ and, similarly, $\|v\|_{\infty} \leq$ $\min \left\{G_{3}, G_{4}, M_{3}, L_{1}\right\}$. Note that $b_{1}<\min \left\{\rho^{-} / p, c_{1}\right\}$ and $b_{2}<$ $\min \left\{\gamma^{-} / q, c_{2}\right\}$. Hence, it follows from (K3), (F1), (I2), (H2), and (66) that

$$
\begin{aligned}
& \mathscr{J}(u, v)=\left.\left.\frac{1}{p} \int_{0}^{T} \rho(t)\right|_{0} ^{c} D_{t}^{\alpha} u(t)\right|^{p} d t+\frac{1}{q} \\
& \cdot \int_{0}^{T} \gamma(t)\left|{ }_{0}^{c} D_{t}^{\beta} v(t)\right|^{q} d t+\sum_{i=1}^{l} I_{i}\left(u\left(t_{i}\right)\right) \\
& +\sum_{j=1}^{m} H_{j}\left(v\left(s_{j}\right)\right)+\int_{0}^{T} K(t, u(t), v(t)) d t \\
& -\int_{0}^{T} F(t, u(t), v(t)) d t \geq \frac{\rho^{-}}{p} \int_{0}^{T}\left|{ }_{0}^{c} D_{t}^{\alpha} u(t)\right|^{p} d t \\
& +\frac{\gamma^{-}}{q} \int_{0}^{T}\left|{ }_{0}^{c} D_{t}^{\alpha} v(t)\right|^{q} d t-d_{7} \sum_{i=1}^{l}\left|u\left(t_{j}\right)\right|^{\theta_{5}} \\
& -d_{8} \sum_{j=1}^{m}\left|v\left(s_{j}\right)\right|^{\theta_{6}}+c_{1} \int_{0}^{T}|u(t)|^{p} d t \\
& +c_{2} \int_{0}^{T}|v(t)|^{q} d t-b_{1} \int_{0}^{T}|u(t)|^{p} d t \\
& -b_{2} \int_{0}^{T}|v(t)|^{q} d t \geq \min \left\{\frac{\rho^{-}}{p}, c_{1}\right\}\|u\|_{p}^{p}
\end{aligned}
$$

$$
\begin{aligned}
& +\min \left\{\frac{\gamma^{-}}{q}, c_{2}\right\}\|v\|_{q}^{q}-d_{7} l\|u\|_{\infty}^{\theta_{5}}-d_{8} m\|v\|_{\infty}^{\theta_{6}} \\
& -b_{1}\|u\|_{p}^{p}-b_{2}\|v\|_{q}^{q} \geq \min \left\{\frac{\rho^{-}}{p}, c_{1}\right\}\|u\|_{p}^{p} \\
& +\min \left\{\frac{\gamma^{-}}{q}, c_{2}\right\}\|v\|_{q}^{q}-d_{7} l C_{\alpha, p, \infty}^{\theta_{5}}\|u\|_{p}^{\theta_{5}} \\
& -d_{8} m C_{\beta, q, \infty}^{\theta_{6}}\|v\|_{q}^{\theta_{6}}-b_{1}\|u\|_{p}^{p}-b_{2}\|v\|_{q}^{q} \\
& \geq \min \left\{\min \left\{\frac{\rho^{-}}{p}, c_{1}\right\}-b_{1}, \min \left\{\frac{\gamma^{-}}{q}, c_{2}\right\}-b_{2}\right\} \\
& \cdot \frac{1}{\max \left\{2^{q^{-1}}, 2^{p-1}\right\}}\left(\|u\|_{p}+\|v\|_{q}\right) \min \{p, q\} \\
& -\min \left\{\min \left\{\frac{\rho^{-}}{p}, c_{1}\right\}-b_{1}, \min \left\{\frac{\gamma^{-}}{q}, c_{2}\right\}-b_{2}\right\} \\
& -d_{7} l C_{\alpha, p, \infty}^{\theta_{5}}\|(u, v)\|_{E}^{\theta_{5}}-d_{8} m C_{\beta, q, \infty}^{\theta_{6}}\|(u, v)\|_{E}^{\theta_{6}} .
\end{aligned}
$$

Since $\theta_{5}, \theta_{6} \in[0, \min \{p, q\}),(77)$ implies that there exists a sufficiently large constant $\rho>0$ such that $\mathcal{J}(u, v)>0$ for all $(u, v) \in E$ with $\|(u, v)\|_{E}=\rho$.

Lemma 22. Assume that (Ad), (K3), (F1), and (IH2)' hold. There are constants $\rho, \xi>0$ such that $\left.\mathscr{F}\right|_{\partial B_{\rho} \cap E} \geq \xi$.

Proof. By (77) and $(\mathrm{IH} 2)^{\prime}$, it is easy to obtain that

$\mathscr{J}(u, v)$

$$
\begin{aligned}
& \geq \min \left\{\min \left\{\frac{\rho^{-}}{p}, c_{1}\right\}-b_{1}, \min \left\{\frac{\gamma^{-}}{q}, c_{2}\right\}-b_{2}\right\} \\
& \cdot \frac{1}{\max \left\{2^{q-1}, 2^{p-1}\right\}}\|(u, v)\|_{E}^{\min \{p, q\}} \\
& -\min \left\{\min \left\{\frac{\rho^{-}}{p}, c_{1}\right\}-b_{1}, \min \left\{\frac{\gamma^{-}}{q}, c_{2}\right\}-b_{2}\right\} \\
& -d_{7}^{\prime} l C_{\alpha, p, \infty}^{\min \{p, q\}}\|(u, v)\|_{E}^{\min \{p, q\}} \\
& -d_{8}^{\prime} m C_{\beta, q, \infty}^{\min \{p, q\}}\|(u, v)\|_{E}^{\min \{p, q\}} .
\end{aligned}
$$

Then (22) and (78) imply that there exists a sufficiently large constant $\rho>0$ such that $\mathscr{J}(u, v)>0$ for all $(u, v) \in E$ with $\|(u, v)\|_{E}=\rho$.

Lemma 23. For each finite dimensional subspace $\widetilde{E} \subset E$, there is $R=R(\widetilde{E})$ such that $\mathscr{J}(u, v) \leq 0$ on $\widetilde{E} \backslash B_{R(\widetilde{E})}$.

Proof. For each given finite dimensional space $\widetilde{E} \subset E$, we claim that there exists $R>0$ such that $\varphi(u) \leq 0$ on $\widetilde{E} / B_{R}$. Indeed, obviously, for any $(u, v) \in \widetilde{E},(u, v)$ can be rewritten by $(u, v)=(u, 0)+(0, v)$, where $u \in \widetilde{E_{1}} \subset E_{0}^{\alpha, p}(0, T), v \in \widetilde{E_{2}} \subset$ $E_{0}^{\beta, q}(0, T)$, and $\widetilde{E_{1}}$ and $\widetilde{E_{2}}$ are finite dimensional ones. So there 
exist positive constants $d_{9}, d_{10}, d_{11}, d_{12}, d_{13}, d_{14}, d_{15}, d_{16}$ such that

$$
\begin{aligned}
& d_{9}\|u\|_{L^{p}}^{p} \leq \int_{0}^{T}|u(t)|^{\max \{p, q\}} d t \leq d_{10}\|u\|_{L^{p}}^{p}, \\
& d_{11}\|v\|_{L^{q}}^{q} \leq \int_{0}^{T}|v(t)|^{\max \{p, q\}} d t \leq d_{12}\|v\|_{L^{q}}^{q}, \\
& d_{13}\|u\|_{p}^{p} \leq \int_{0}^{T}|u(t)|^{p} d t \leq d_{14}\|u\|_{p}^{p}, \\
& d_{15}\|v\|_{q}^{q} \leq \int_{0}^{T}|v(t)|^{q} d t \leq d_{16}\|v\|_{q}^{q} .
\end{aligned}
$$

Similar to the proof of Theorem 1.1 in [4], by (K2) and assumption $(W 0)$, there exists positive constant $D_{0}$ such that

$$
\begin{aligned}
K(t, x, y) \leq & D_{0} b(t)|x|^{\max \{p, q\}}+D_{0} b(t)|y|^{\max \{p, q\}} \\
& +D_{0} b(t)
\end{aligned}
$$

for all $(x, y) \in \mathbb{R}^{N} \times \mathbb{R}^{N}$ and a.e. $t \in[0, T]$. By $(F 2)$, there exist

$$
\begin{aligned}
D_{1} & >\max \left\{\frac { 1 } { d _ { 1 3 } } \left(\frac{\operatorname{esssup}_{[0, T]} \rho(t)}{p}+D_{0}\right.\right. \\
\cdot & \left.\operatorname{esssup}_{[0, T]} b(t) \cdot d_{10}+d_{3} l C_{\alpha, p, \infty}^{p}\right), \\
& \frac{1}{d_{15}}\left(\frac{\operatorname{essup}_{[0, T]} \gamma(t)}{q}+D_{0} \cdot \operatorname{essup}_{[0, T]} b(t) \cdot d_{12}\right. \\
& \left.\left.+d_{5} m C_{\beta, q, \infty}^{q}\right)\right\}
\end{aligned}
$$

and positive constant $M_{4}$ such that

$$
F(t, x, y) \geq D_{1}|x|^{p}+D_{1}|y|^{q}
$$

for all $(x, y) \in \mathbb{R}^{N} \times \mathbb{R}^{N}$ with $|x|+|y| \geq M_{4}$ and a.e. $t \in$ $[0, T]$. Then by $(82)$ and assumption $(W 0)$, there exist positive constants $D_{2}$ and $D_{3}$ such that

$$
F(t, x, y) \geq D_{1}|x|^{p}+D_{0}|y|^{q}-D_{2}-D_{3} b(t)
$$

for all $(x, y) \in \mathbb{R}^{N} \times \mathbb{R}^{N}$ and a.e. $t \in[0, T]$. By $(I 1)$ and $(H 1)$, there exist positive constants $D_{4}, D_{5}$ such that

$$
\begin{gathered}
I_{i}(x) \leq d_{3}|x|^{p}+D_{4} b(t), \\
H_{j}(y) \leq d_{5}|y|^{q}+D_{5} b(t)
\end{gathered}
$$

for all $(x, y) \in \mathbb{R}^{N} \times \mathbb{R}^{N}$ and a.e. $t \in[0, T]$. It follows from (83), (80), (84), and (79) that

$$
\begin{gathered}
\mathscr{J}(u, v)=\left.\left.\frac{1}{p} \int_{0}^{T} \rho(t)\right|_{0} ^{c} D_{t}^{\alpha} u(t)\right|^{p} d t+\frac{1}{q} \\
\cdot \int_{0}^{T} \gamma(t)\left|{ }_{0}^{c} D_{t}^{\alpha} v(t)\right|^{q} d t+\sum_{i=1}^{l} I_{i}\left(u\left(t_{j}\right)\right) \\
+\sum_{j=1}^{m} H_{j}\left(v\left(s_{j}\right)\right)+\int_{0}^{T} K(t, u(t), v(t)) d t
\end{gathered}
$$

$-\int_{0}^{T} F(t, u(t), v(t)) d t \leq \frac{\operatorname{esssup}_{[0, T]} \rho(t)}{p}$

$\cdot \int_{0}^{T}\left|{ }_{0}^{c} D_{t}^{\alpha} u(t)\right|^{p} d t+\frac{\operatorname{esssup}_{[0, T]} \gamma(t)}{q}$

$\cdot \int_{0}^{T}\left|{ }_{0}^{c} D_{t}^{\alpha} v(t)\right|^{q} d t+d_{3} \sum_{i=1}^{l}\left|u\left(t_{j}\right)\right|^{p}$

$+d_{5} \sum_{j=1}^{m}\left|v\left(s_{j}\right)\right|^{q}+D_{0} \int_{0}^{T} b(t)|u(t)|^{\max \{p, q\}} d t$

$+D_{0} \int_{0}^{T} b(t)|v(t)|^{\max \{p, q\}} d t-D_{1} \int_{0}^{T}|u(t)|^{p} d t$

$-D_{1} \int_{0}^{T}|v(t)|^{q} d t+D_{2} T+\left(D_{3}+D_{0}\right)$

$\cdot \int_{0}^{T} b(t) d t+D_{4} l+D_{5} m \leq \frac{\operatorname{esssup}_{[0, T]} \rho(t)}{p}$

$\cdot \int_{0}^{T}\left|{ }_{0}^{c} D_{t}^{\alpha} u(t)\right|^{p} d t+\frac{\operatorname{esssup}_{[0, T]} \gamma(t)}{q}$

$\cdot \int_{0}^{T}\left|{ }_{0}^{c} D_{t}^{\alpha} v(t)\right|^{q} d t+d_{3} l\|u\|_{\infty}^{p}+d_{5} m\|v\|_{\infty}^{q}+D_{0}$

$\cdot \operatorname{essup}_{[0, T]} b(t) \cdot d_{10} \int_{0}^{T}|u(t)|^{p} d t+D_{0}$

$\cdot \operatorname{esssup}_{[0, T]} b(t) \cdot d_{12} \int_{0}^{T}|v(t)|^{q} d t-D_{1} d_{13}\|u\|_{p}^{p}$

$-D_{1} d_{15}\|v\|_{q}^{q}+D_{2} T+\left(D_{3}+D_{0}\right) \int_{0}^{T} b(t) d t+D_{4} l$

$+D_{5} m \leq\left(\frac{\operatorname{esssup}_{[0, T]} \rho(t)}{p}+D_{0} \cdot \operatorname{esssup}_{[0, T]} b(t)\right.$

$\left.\cdot d_{10}\right)\|u\|_{p}^{p}+\left(\frac{\operatorname{esssup}_{[0, T]} \gamma(t)}{q}+D_{0}\right.$

$\left.\cdot \operatorname{esssup}_{[0, T]} b(t) \cdot d_{12}\right)\|v\|_{q}+d_{3} l C_{\alpha, p, \infty}^{p}\|u\|_{p}^{p}$

$+d_{5} m C_{\beta, q, \infty}^{q}\|v\|_{q}^{q}-D_{1} d_{13}\|u\|_{p}^{p}-D_{1} d_{15}\|v\|_{q}^{q}$

$+D_{2} T+\left(D_{3}+D_{0}\right) \int_{0}^{T} b(t) d t+D_{4} l+D_{5} m$

$=\left(\frac{\operatorname{esssup}_{[0, T]} \rho(t)}{p}+D_{0} \cdot \operatorname{esssup}_{[0, T]} b(t) \cdot d_{10}\right.$

$\left.+d_{3} l C_{\alpha, p, \infty}^{p}-D_{1} d_{13}\right)\|u\|_{p}^{p}+\left(\frac{\operatorname{esssup}_{[0, T]} \gamma(t)}{q}\right.$

$\left.+D_{0} \cdot \operatorname{essup}_{[0, T]} b(t) \cdot d_{12}+d_{5} m C_{\beta, q, \infty}^{q}-D_{1} d_{15}\right)$

$\cdot\|v\|_{q}^{q}+D_{2} T+\left(D_{3}+D_{0}\right) \int_{0}^{T} b(t) d t+D_{4} l$

$+D_{5} m$. 
Thus, (81) and the above inequality imply that there exists a sufficiently large constant $R>0$ such that $\mathscr{J}(u, v)<0$ if $\|u\|_{p}=\|v\|_{q}=R / 2$.

Proof of Theorems 1 and 2. By (W1), (I3), and (H3), we have $\mathscr{J}(0,0)=0$ and $\mathscr{J}$ is even. Similar to the proof of Lemma 4.4 in [44], we can choose $e_{j} \in E \backslash\{0\}, j=1,2, \ldots$, and define $V=\operatorname{span}\left\{e_{1}\right\}$ and $X=\overline{\operatorname{span}\left\{e_{j}, j=2, \ldots\right\}}$. Then $E=V \oplus X$. Note that $\partial B_{\rho} \cap X \subset \partial B_{\rho} \cap E$. Thus by Lemma 21 (or Lemma 22) and Lemma 23 it is easy to see that (i) and (ii) of Lemma 18 hold. Lemma 20 implies that $\mathscr{J}$ satisfies Cerami condition. So, by Lemma 18 and Remark 19 , there exists a sequence $\left\{\left(u_{n}, v_{n}\right)\right\}$ such that $\mathscr{J}\left(u_{n}, v_{n}\right) \rightarrow+\infty$ and then it is easy to see that $\left\|\left(u_{n}, v_{n}\right)\right\| \rightarrow \infty$ as $n \rightarrow \infty$.

\section{Conflicts of Interest}

The authors declare that they have no conflicts of interest.

\section{Acknowledgments}

This project is supported by the National Natural Science Foundation of China (no. 11226135 and no. 11301235).

\section{References}

[1] J. Mawhin and M. Willem, Critical Point Theory and Hamiltonian Systems, vol. 74, Springer, New York, NY. USA, 1989.

[2] P. H. Rabinowitz, Minimax Methods in Critical Point Theory with Applications to Differential Equations, vol. 65, American Mathematical Society, Providence, RI, USA, 1986.

[3] Y. H. Ding, Variational Methods for Strongly Indefinite Problems, World Scientific Publishing Co. Pte. Ltd., Hackensack, NJ, 2008.

[4] X. Yang and H. Chen, "Periodic solutions for a nonlinear $(q, p)$ Laplacian dynamical system with impulsive effects," Applied Mathematics and Computation, vol. 40, no. 1-2, pp. 607-625, 2012.

[5] X. Zhang and X. Tang, "Subharmonic solutions for a class of non-quadratic second order Hamiltonian systems," Nonlinear Analysis: Real World Applications, vol. 13, no. 1, pp. 113-130, 2012.

[6] X. H. Tang and J. Jiang, "Existence and multiplicity of periodic solutions for a class of second-order Hamiltonian systems," Computers \& Mathematics with Applications, vol. 59, no. 12, pp. 3646-3655, 2010.

[7] X. Tang and J. Chen, "Infinitely many homoclinic orbits for a class of discrete Hamiltonian systems," Advances in Difference Equations, vol. 1, pp. 1-12, 2013.

[8] F. Zhao and Y. Ding, "Infinitely many solutions for a class of nonlinear Dirac equations without symmetry," Nonlinear Analysis. Theory, Methods \& Applications, vol. 70, no. 2, pp. 921935, 2009.

[9] G. Li, X. Luo, and W. Shuai, "Sign-changing solutions to a gauged nonlinear Schrödinger equation," Journal of Mathematical Analysis and Applications, vol. 455, no. 2, pp. 1559-1578, 2017.

[10] Z. Zhang and K. Perera, "Sign changing solutions of Kirchhoff type problems via invariant sets of descent flow," Journal of Mathematical Analysis and Applications, vol. 317, no. 2, pp. 456463, 2006.

[11] Y. Ye and C.-L. Tang, "Existence and multiplicity of solutions for Schr? dinger CPoisson equations with sign-changing potential,"
Calculus of Variations and Partial Differential Equations, vol. 53, no. 1-2, pp. 383-411, 2015.

[12] F. Jiao and Y. Zhou, "Existence of solutions for a class of fractional boundary value problems via critical point theory," Computers \& Mathematics with Applications, vol. 62, no. 3, pp. 1181-1199, 2011.

[13] P. Li, H. Wang, and Z. Li, "Infinitely many solutions to boundary value problems for a coupled system of fractional differential equations," Journal of Nonlinear Sciences and Applications. JNSA, vol. 9, no. 5, pp. 3433-3444, 2016.

[14] Y. Zhao, H. Chen, and B. Qin, "Multiple solutions for a coupled system of nonlinear fractional differential equations via variational methods," Applied Mathematics and Computation, vol. 257, pp. 417-427, 2015.

[15] C. Torres, "Mountain pass solution for a fractional boundary value problem," Journal of Fractional Calculus and Applications, vol. 5, no. 1, pp. 1-10, 2014.

[16] Y. Li, H. Sun, and Q. Zhang, "Existence of solutions to fractional boundary-value problems with a parameter," Electronic Journal of Differential Equations, vol. 2013, no. 141, 12 pages, 2013.

[17] J. Chen and X. H. Tang, "Existence and multiplicity of solutions for some fractional boundary value problem via critical point theory," Abstract and Applied Analysis, vol. 2012, Article ID 648635, 21 pages, 2012.

[18] G. Bonanno and S. A. Marano, "On the structure of the critical set of non-differentiable functions with a weak compactness condition," Applicable Analysis: An International Journal, vol. 89, no. 1, pp. 1-10, 2010.

[19] J. J. Nieto and D. O’Regan, "Variational approach to impulsive differential equations," Nonlinear Analysis: Real World Applications, vol. 10, no. 2, pp. 680-690, 2009.

[20] Y. Tian and W. Ge, "Variational methods to Sturm-Liouville boundary value problem for impulsive differential equations," Nonlinear Analysis. Theory, Methods \& Applications, vol. 72, no. 1, pp. 277-287, 2010.

[21] Y. Tian and W. Ge, "Applications of variational methods to boundary-value problem for impulsive differential equations," Proceedings of the Edinburgh Mathematical Society, vol. 51, no. 2, pp. 509-527, 2008.

[22] J. Sun, H. Chen, J. J. Nieto, and M. Otero-Novoa, "The multiplicity of solutions for perturbed second-order Hamiltonian systems with impulsive effects," Nonlinear Analysis. Theory, Methods \& Applications, vol. 72, no. 12, pp. 4575-4586, 2010.

[23] G. D' Aguì, B. Di Bella, and S. Tersian, "Multiplicity results for superlinear boundary value problems with impulsive effects," Mathematical Methods in the Applied Sciences, vol. 39, no. 5, pp. 1060-1068, 2016.

[24] J. Zhou and Y. Li, "Existence and multiplicity of solutions for some Dirichlet problems with impulsive effects," Nonlinear Analysis. Theory, Methods \& Applications, vol. 71, no. 7-8, pp. 2856-2865, 2009.

[25] Z. Zhang and R. Yuan, "An application of variational methods to Dirichlet boundary value problem with impulses," Nonlinear Analysis: Real World Applications, vol. 11, no. 1, pp. 155-162, 2010.

[26] J. Li and H. Chen, "Variational approach to impulsive differential equations with dirichlet boundary conditions," Boundary Value Problems, vol. 2010, Article ID 325415, 2010.

[27] G. Bonanno, R. Rodríguez-López, and S. Tersian, "Existence of solutions to boundary value problem for impulsive fractional differential equations," Fractional Calculus and Applied Analysis, vol. 17, no. 3, pp. 717-744, 2014. 
[28] R. Rodriguez-López and S. Tersian, "Multiple solutions to boundary value problem for impulsive fractional differential equations," Fractional Calculus and Applied Analysis, vol. 17, no. 4, pp. 1016-1038, 2014.

[29] N. Nyamoradi and R. Rodríguez-López, "On boundary value problems for impulsive fractional differential equations," Applied Mathematics and Computation, vol. 271, pp. 874-892, 2015.

[30] Y. Zhao and Y. Zhao, "Nontrivial solutions for a class of perturbed fractional differential systems with impulsive effects," Boundary Value Problems, Paper No. 129, 16 pages, 2016.

[31] G. Bonanno, "A critical point theorem via the Ekeland variational principle," Nonlinear Analysis. Theory, Methods \& Applications, vol. 75, no. 5, pp. 2992-3007, 2012.

[32] S. Heidarkhani, Y. Zhao, G. Caristi, G. A. Afrouzi, and S. Moradi, "Infinitely many solutions for perturbed impulsive fractional differential systems," Applicable Analysis: An International Journal, vol. 96, no. 8, pp. 1401-1424, 2017.

[33] G. Bonanno and G. M. Bisci, "Infinitely many solutions for a boundary value problem with discontinuous nonlinearities," Boundary Value Problems, vol. 2009, Article ID 670675, 2009.

[34] Y. Zhao, H. Chen, and C. Xu, "Nontrivial solutions for impulsive fractional differential equations via Morse theory," Applied Mathematics and Computation, vol. 307, pp. 170-179, 2017.

[35] Y. Zhao and L. Tang, "Multiplicity results for impulsive fractional differential equations with p-Laplacian via variational methods," Boundary Value Problems, vol. 2017, no. 213, 2017.

[36] E. Zeidler, Nonlinear Functional Analysis and Its Applications, Springer, New York, NY, USA, 1985.

[37] X. Yang, "Existence and multiplicity of weak solutions for a nonlinear impulsive (q, p) -Laplacian dynamical system," Advances in Difference Equations, vol. 2017, no. 1, article no. 128, 2017.

[38] X. Yang and H. Chen, "Periodic solutions for $(q, p)$-Laplacian autonomous system with impulsive effects," Journal of Applied Mathematics, vol. 2011, Article ID 378389, 19 pages, 2011.

[39] I. Podlubny, Fractional Differential Equations, Academic Press, New York, NY, USA, 1993.

[40] A. A. Kilbas, H. M. Srivastava, and J. J. Trujillo, "Preface," NorthHolland Mathematics Studies, vol. 204, pp. 7-10, 2006.

[41] K. Diethelm, The Anaysis of Fractional Differential Equations, Springer, Heidelberg, 2010.

[42] Y. Zhou, Basic Theory of Fractional Differential Equations, World Scientific Publishing Co. Pte. Ltd., Hackensack, NJ, 2014.

[43] P. Bartolo, V. Benci, and D. Fortunato, "Abstract critical point theorems and applications to some nonlinear problems with strong resonance at infinity," Nonlinear Analysis, vol. 7, no. 9, pp. 981-1012, 1983.

[44] L. Wang, X. Zhang, and H. Fang, "Existence and multiplicity of solutions for a class of quasilinear elliptic systems in OrliczSobolev spaces," Journal of Nonlinear Sciences and Applications. JNSA, vol. 10, no. 7, pp. 3792-3814, 2017. 


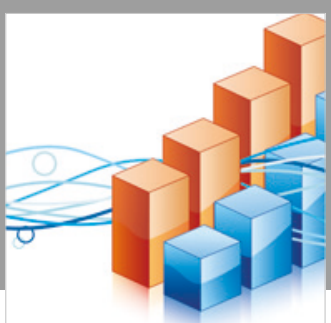

Advances in

Operations Research

\section{-n-m}
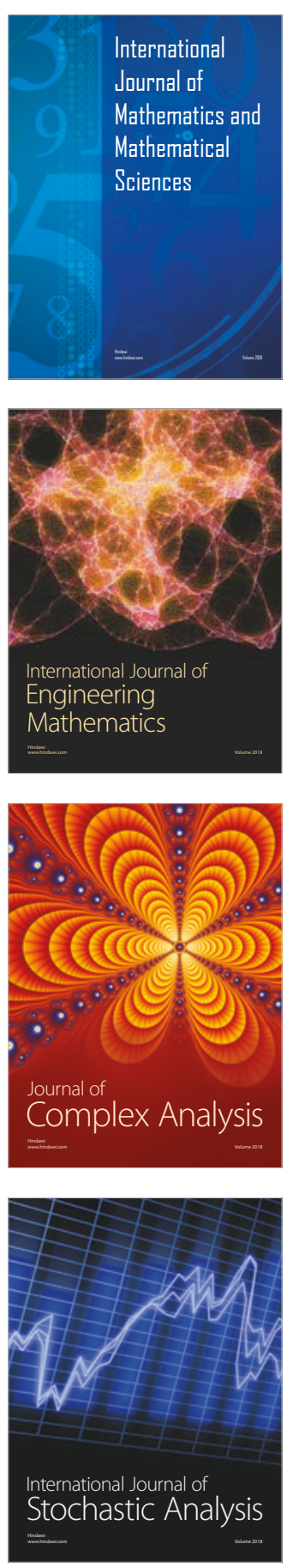
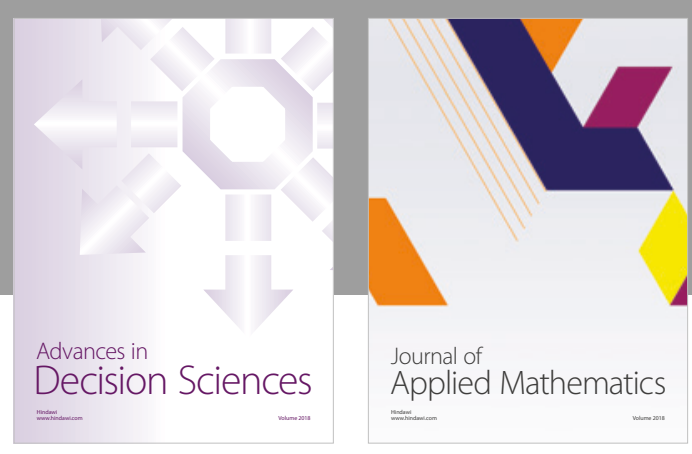

Journal of

Applied Mathematics
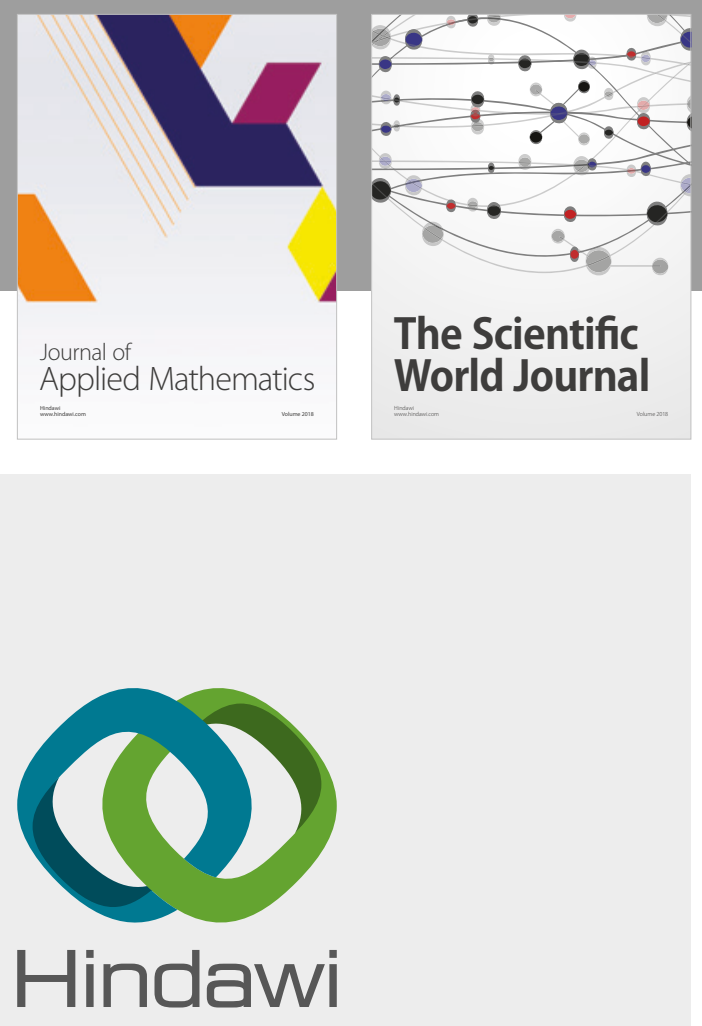

Submit your manuscripts at

www.hindawi.com

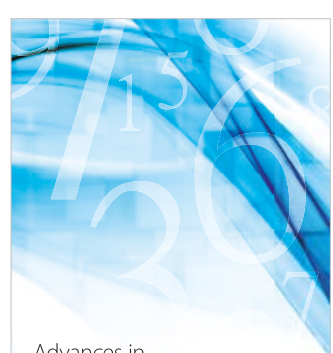

Advances in
Numerical Analysis
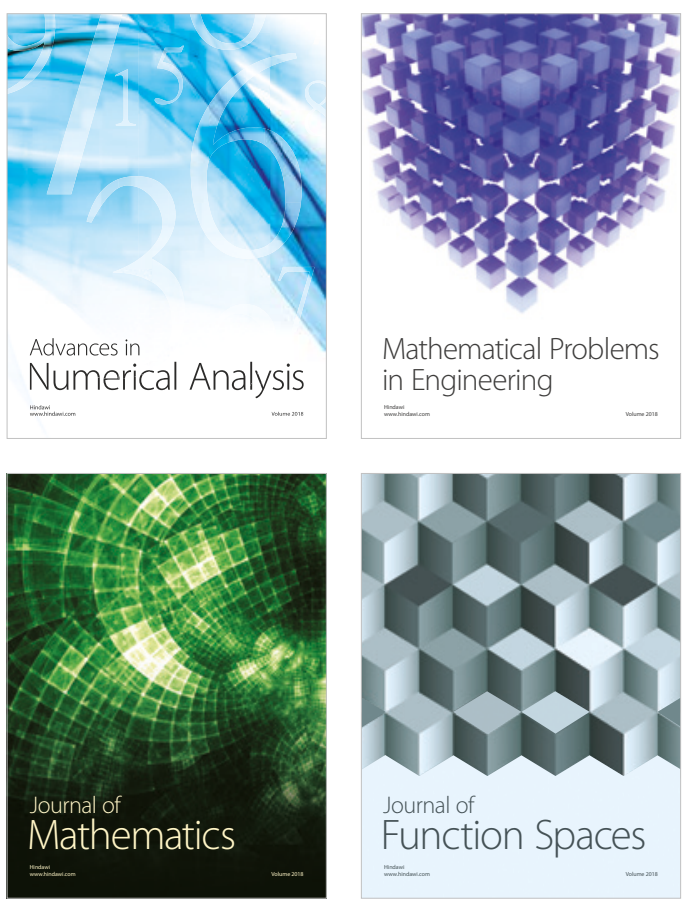

Mathematical Problems in Engineering



International Journal of

Differential Equations

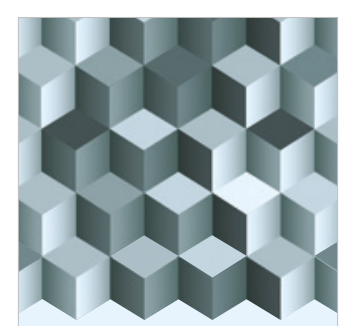

Journal of

Function Spaces

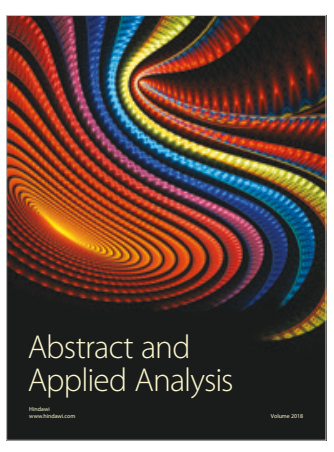

The Scientific

World Journal

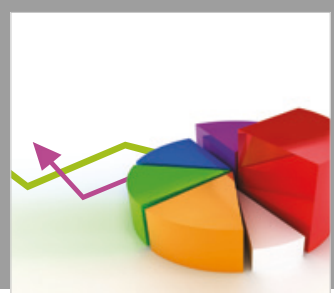

Journal of

Probability and Statistics
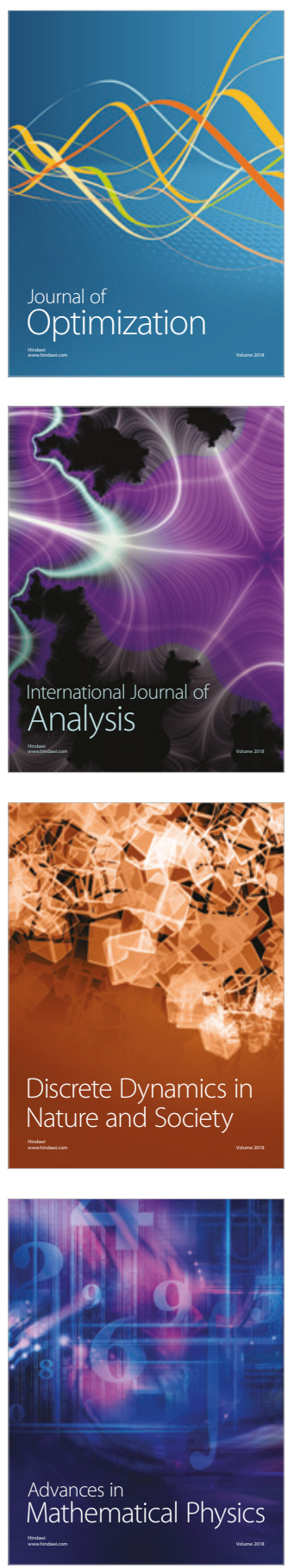